Discussion Paper Series A No.473

\title{
Axiomatic Bargaining Theory on Opportunity Assignments
}

\author{
Yongsheng $\mathrm{Xu}$ \\ (Department of Economics, Andrew Young School of Policy Studies, \\ Georgia State University) \\ and \\ Naoki Yoshihara \\ (The Institute of Economic Research, Hitotsubashi University)
}

J anuary 2006

The Institute of Economic Research

Hitotsubashi University

Kunitachi, Tokyo, 186-8603 J apan 
Axiomatic bargaining theory on opportunity assignments*

\author{
Yongsheng $\mathrm{Xu}$ \\ Department of Economics \\ Andrew Young School of Policy Studies \\ Georgia State University \\ Atlanta, GA 30303, U.S.A. \\ Email: yxu3@gsu.edu \\ and
}

China Academy of Public Finance and Public Policy

Central University of Finance and Economics

Beijing, China

\author{
Naoki Yoshihara \\ Institute of Economic Research \\ Hitotsubashi University \\ 2-4 Naka, Kunitachi \\ Tokyo, Japan 186-8603
}

Email: yosihara@ier.hit-u.ac.jp

December, 2005, This version November 2006

\footnotetext{
*Financial supports from the Department of Economics, Andrew Young School of Policy Studies, Georgia State University, and from the Ministry of Education, Culture, Sports, Science and Technology of Japan are gratefully acknowledged. We also thank Atsushi Kajii, Chiaki Hara, Shinsuke Nakamura, and audiences of the 8th international meeting of the Society for Social Choice and Welfare held at Istanbul in July 2006, Keio University, Kyoto University, and the 12th Decentralization Conference in Japan held at Osaka Prefecture University in October 2006 where this paper was presented for their valuable comments.
} 


\begin{abstract}
This paper discusses issues of axiomatic bargaining problems over opportunity assignments. The fair arbitrator uses the principle of "equal opportunity" for all players to make the recommendation on resource allocations. A framework in such a context is developed and several classical solutions to standard bargaining problems are reformulated and axiomatically characterized.
\end{abstract}

JEL Classification Numbers: C71, C78, D60, D63, D70

Keywords: Opportunity sets, bargaining over opportunity assignments, Nash solution, egalitarian solution, Kalai-Smorodinsky solution 


\section{Introduction}

In standard axiomatic bargaining models originated from Nash (1950), a typical interpretation of the solution to bargaining problems is the recommendation made by a "fair arbitrator" such as the Judge in civil trials, or the function of Dispute Settlement Body in the WTO mechanism, etc. In such models, the recommendation is based solely on players' utilities. In many contexts, however, the "fair arbitrator" may have other principles in mind when making a recommendation.

For instance, consider the distribution issue of a father's inheritance among his children. The father, as a "fair arbitrator," may have the principle of "equal opportunities" for his children and would like to distribute his wealth among his children giving them equal opportunities to do well in their respective lives. Likewise, when educational resources are to be allocated among local public schools, the local government's board of education, as the "fair arbitrator," may propose an allocation that "equalizes" school children's opportunity sets for future jobs, skills, college admissions, lives, etc. In both settings of the above examples, each recommendation of a resource allocation by the "fair arbitrator" effectively identifies a profile of "opportunities" or opportunity sets for individuals involved. The crucial difference from standard axiomatic bargaining models in these examples is that the recommendation made by the arbitrator is not based on utilities of the individuals involved, but on opportunity sets that the recommended resource allocation may give rise to the involved individuals.

This departure from considerations of utilities of individuals to concerns of opportunity sets of individuals is well in line with the recent literature on opportunities and equality of opportunities. One stream of the literature is in political philosophy such as Sen (1980, 1985), Arneson (1989), and Cohen (1993), while the other stream is in economics, see, for example, Sen (2002), Pattanaik and Xu (1990), Kranich (1996), and Herrero (1997). In the latter stream of the literature, each individual is characterized by his opportunity sets, from which his well-being or welfare is evaluated.

An opportunity set of an individual is interpreted as a set of feasible options or alternatives available to the individual for living a life. Depending on the context, those alternatives can be commodity bundles, or bundles of characteristics à la Lancaster and Gorman, or bundles of functionings à la Sen (1980, 1985), and Nussbaum $(1988,1993,2000)$. A resource allocation in an economy then identifies a collection of opportunity sets, one for each indi- 
vidual in the economy. Note that, for a given resource allocation, opportunity sets of individuals are necessarily interdependent. Note also that different resource allocations can give rise to various collections of opportunity sets for the individuals in the economy.

The question that we want to have an answer in this paper is the following. Among various collections of opportunity sets for the individuals involved, how should the "fair arbitrator" make the recommendation on a resource allocation that yields a profile of opportunity sets for individuals in the economy deemed as "fair"? For this purpose, we extend standard bargaining models to the setting in which each individual is endowed with his opportunity sets, which are generated by his consumption bundles given his individual characteristics, and, in which the fair arbitrator makes recommendations based on profiles of opportunity sets for the individuals in the economy. ${ }^{1}$ In particular, we reformulate the three classical solutions, the Nash, the egalitarian, and the Kalai-Smorodinsky, in our context and study them axiomatically.

The remaining of the paper is organized as follows. In Section 2, we introduce our economic environments and our problem. Section 3 presents some preliminary properties on the social evaluation of opportunity sets and defines the three solutions, the Nash, the egalitarian, and the Kalai-Smorodinsky, in our context. Section 4 presents the axioms used for our characterization results. In Section 5, we axiomatically characterize and present some interpretations for the three solutions. We conclude the paper in Section 5 by briefly commenting on our approach and the results.

\section{Economic environments and bargaining prob- lems on opportunity assignments}

There are (possibly) infinitely many types of goods (commodities). The universe of "potential goods" is denoted by $\Xi$, and the class of non-empty and finite subsets of $\Xi$ is designated by $\mathcal{M}$, with generic elements, $K, L$, $M, \ldots$, . The cardinality of $M \in \mathcal{M}$ is denoted by $\# M$. Given $M \in \mathcal{M}$, let $\mathbb{R}_{+}^{m}$, where $m=\# M$, designate the Cartesian product of $\# M$ copies of $\mathbb{R}_{+}$.

\footnotetext{
${ }^{1}$ Gotoh and Yoshihara (2003) discuss allocation mechanisms which assign individuals capability sets through distributing outputs produced by them. Their approach is quite different from the approach based on bargaining that this paper addresses.
} 
For each $M \in \mathcal{M}$, let us denote generic commodity bundle in $\mathbb{R}_{+}^{m}$ by $x \in \mathbb{R}_{+}^{m}$.

The population in the economy is given by the set $N=\{1, \cdots, n\}$, where $2 \leq n<+\infty$. Given a list of commodities $M \in \mathcal{M}$, every individual has a common consumption space $\mathbb{R}_{+}^{m}$. There are $k$ basic living conditions in the economy, which are relevant for all individuals for the purpose of describing their objective well-beings attainable by means of their consumption vectors. These basic living conditions can be interpreted broadly. For example, they can be skills that individuals can develop through education, or they can be occupations which individual can engage in after the graduation of school. Or they can be characteristics of commodities in the sense of Gorman (1980) and Lancaster (1966), or they can be various functionings according to Sen (1980, $1985)$ and Nussbaum $(1988,1993,2000)$. For our formal analysis, we do not need to stick to a particular interpretation though a certain interpretation may be more appropriate than other interpretations for a given context.

Thus, an achievement of living condition $f$, where $f=1,2, \cdots, k$, by individual $i$ is denoted by $b_{i f} \in \mathbb{R}_{+}$. Individual $i$ 's achievement of basic living conditions is given by listing $b_{i f}: \mathbf{b}_{i}=\left(b_{i 1}, \cdots, b_{i k}\right) \in \mathbb{R}_{+}^{k}$. Given $M \in \mathcal{M}$, each individual $i \in N$ can be characterized by his opportunity correspondence $c_{i}^{m}: \mathbb{R}_{+}^{m} \rightarrow \mathbb{R}_{+}^{k}$ which associates to every consumption vector $x_{i} \in \mathbb{R}_{+}^{m}$ a non-empty subset $c_{i}^{m}\left(x_{i}\right)$ of $\mathbb{R}_{+}^{k}$. The intended interpretation is that $i$ is able to have access to each living-condition vector $\mathbf{b}_{i} \in c_{i}^{m}\left(x_{i}\right)$ by means of his consumption vector $x_{i}$. Each opportunity correspondence satisfies the following requirements:

(a) For all $x_{i}, x_{i}^{\prime} \in \mathbb{R}_{+}^{m}$ such that $x_{i} \leq x_{i}^{\prime}, c_{i}^{m}\left(x_{i}\right) \subseteq c_{i}^{m}\left(x_{i}^{\prime}\right)$ hold; ${ }^{2}$

(b) For some $x_{i} \in \mathbb{R}_{+}^{m} \backslash\{\mathbf{0}\}, c_{i}^{m}\left(x_{i}\right) \cap \mathbb{R}_{++}^{k} \neq \varnothing$ holds, and $c_{i}^{m}(\mathbf{0})=\{\mathbf{0}\}$;

(c) For all $x_{i} \in \mathbb{R}_{+}^{m}, c_{i}^{m}\left(x_{i}\right)$ is compact and comprehensive in $\mathbb{R}_{+}^{k}$; and

(d) $c_{i}^{m}$ is continuous on $\mathbb{R}_{+}^{k}$.

Let $\mathfrak{C}^{M}$ be the set of all possible opportunity correspondences defined on $\mathbb{R}_{+}^{m}$, which satisfy the above (a), (b),(c) and (d). Given $M \in \mathcal{M}$, an economy with $\bar{x}$ endowments of $M$-goods is described by a list $\mathbf{e}=\left(M, \mathbf{c}^{m}, \bar{x}\right)=$ $\left(M,\left(c_{i}^{m}\right)_{i \in N}, \bar{x}\right)$, where $M \in \mathcal{M}, \mathbf{c}^{m} \in \mathfrak{C}^{M n}, \bar{x} \in \mathbb{R}_{+}^{m}$, and $\mathfrak{C}^{M n}$ stands for the $n$-fold Cartesian product of $\mathfrak{C}^{M}$. Let $\mathcal{E}^{M}$ be the class of all such economies with $\bar{x}$ endowments of $M$-goods. Let $\mathcal{E} \equiv \underset{M \in \mathcal{M}}{\cup} \mathcal{E}^{M}$. Given $\mathbf{e}=\left(M, \mathbf{c}^{m}, \bar{x}\right) \in$

\footnotetext{
${ }^{2}$ For all vectors $\mathbf{a}=\left(a_{1}, \ldots, a_{p}\right)$ and $\mathbf{b}=\left(b_{1}, \ldots, b_{p}\right) \in \mathbb{R}^{p}, \mathbf{a} \geq \mathbf{b}$ if and only if $a_{i} \geq b_{i}$ $(i=1, \ldots, p) ; \mathbf{a}>\mathbf{b}$ if and only if $\mathbf{a} \geq \mathbf{b}$ and $\mathbf{a} \neq \mathbf{b} ; \mathbf{a} \gg \mathbf{b}$ if and only if $a_{i}>b_{i}$ $(i=1, \ldots, p)$.
} 
$\mathcal{E}^{M}$, a vector $\mathbf{x}=\left(x_{i}\right)_{i \in N} \in \mathbb{R}_{+}^{m n}$ is feasible for $\mathbf{e} \in \mathcal{E}^{M}$ if for all $i \in N$, $x_{i} \in \mathbb{R}_{+}^{m}$, and $\sum x_{i} \leq \bar{x}$. We denote by $A(\mathbf{e})$ the set of feasible allocations for $\mathbf{e} \in \mathcal{E}^{M}$. Let $A(\mathcal{E}) \equiv \cup_{\mathbf{e} \in \mathcal{E}} A(\mathbf{e})$.

For each individual $i \in N$, given $M \in \mathcal{M}$ and given $i$ 's consumption vector $x_{i}, c_{i}^{m}\left(x_{i}\right)$ generates a opportunity set $C_{i}=c_{i}^{m}\left(x_{i}\right)$ for $i$. An opportunity assignment is a list of $n$ opportunity sets one for each individual in the society. Given $\mathbf{e}=\left(M, \mathbf{c}^{m}, \bar{x}\right) \in \mathcal{E}$, the set of possible opportunity assignments for $\mathbf{e} \in \mathcal{E}$ is:

$\mathcal{C}(\mathbf{e}) \equiv\left\{\mathbf{C}=\left(C_{i}\right)_{i \in N} \subseteq \mathbb{R}_{+}^{k n} \mid \exists \mathbf{x}=\left(x_{i}\right)_{i \in N} \in A(\mathbf{e}): C_{i}=c_{i}^{m}\left(x_{i}\right)(\forall i \in N)\right\}$.

Note that for any $\mathbf{e}=\left(M, \mathbf{c}^{m}, \bar{x}\right) \in \mathcal{E}$, any $\mathbf{C}=\left(C_{i}\right)_{i \in N} \in \mathcal{C}(\mathbf{e})$, and any $i \in N$, the opportunity $C_{i}$ is a compact, comprehensive set in $\mathbb{R}_{+}^{k}$ containing the origin. For each $i \in N$ and each living condition $f=$ $1, \ldots, k$, let $\max _{f}\left(C_{i}\right)$ be the maximum amount of living condition $f$ by $i$ that he can achieve under his opportunity set $C_{i}$; that is, $\max _{f}\left(C_{i}\right) \equiv$ $\max \left\{b_{f} \mid\left(b_{1}, \cdots, b_{f}, \cdots, b_{k}\right) \in C_{i}\right\}$. Let $\Sigma \equiv\{\mathcal{C} \mid \exists \mathbf{e} \in \mathcal{E}: \mathcal{C}=\mathcal{C}(\mathbf{e})\}$ be the class of all such possible sets of opportunity assignments. Note that each set $\mathcal{C}$ in $\Sigma$ is compact in terms of Hausdorff metric by the assumption (d) of the opportunity correspondence and the fact that $A(\mathbf{e})$ is compact for every $\mathbf{e} \in \mathcal{E}$. Also, for any $\mathcal{C} \in \Sigma$, if $\mathbf{C}=\left(C_{i}\right)_{i \in N} \in \mathcal{C}$, then for each $j \in N$, every living condition $f=1, \ldots, k$, and any $b_{f} \leq \max _{f}\left(C_{j}\right)$, there exists $\mathbf{C}^{\prime}=\left(C_{j}^{\prime}, \mathbf{C}_{-j}\right) \in \mathcal{C}$ such that $b_{f}=\max _{f}\left(C_{j}^{\prime}\right)$ and $C_{j}^{\prime} \subseteq C_{j}$ by the assumption of (a), (b), and (d) of opportunity correspondences.

Let $\mathcal{K}$ be the universal class of compact, comprehensive subsets in $\mathbb{R}_{+}^{k}$ containing the origin. Thus, $C \in \mathcal{K}$ implies that for any $M \in \mathcal{M}$, there exists $c^{m} \in \mathfrak{C}^{M}$ such that for some $x \in \mathbb{R}_{+}^{m}, c^{m}(x)=C$. Note that for each $\mathcal{C} \in \Sigma$ and every $i \in N$, there exists $C_{i}^{*} \in \mathcal{K}$ such that for every $\mathbf{C} \in \mathcal{C}, C_{i}^{*} \supseteq$ $C_{i}$ holds, and $\left(C_{i}^{*}, \mathbf{C}_{-i}^{0}\right) \in \mathcal{C}$ with $C_{j}^{0} \equiv\{\mathbf{0}\}$ for any $j \neq i$. This is followed from the requirement (a) of opportunity correspodences and the definition of $\Sigma$. Given $\mathcal{C} \in \Sigma$, let us denote such $C_{i}^{*}$ by $m_{i}(\mathcal{C})$ for each $i \in N$.

The formal problem that we are interested in is the bargaining problem over opportunity assignments among individuals. Analogous to the standard bargaining model, we can interpret each $\mathcal{C} \in \Sigma$ as a bargaining problem, and a solution to the problem is to pick up a subset of opportunity assignments $\left\{\mathbf{C}=\left(C_{i}\right)_{i \in N}\right\}$ from $\mathcal{C}$. Then, a bargaining solution in this context is a correspondence $F$ which associates to every $\mathcal{C} \in \Sigma$, a non-empty subset $F(\mathcal{C}) \subseteq \mathcal{C}$ 
How is our model related to the motivation discussed in the Introduction? The following examples may help us in understanding our approach.

Example 1: Let $k$ be the number of skills that an individual can develop through education, and let $x \in \mathbf{R}_{+}^{m}$ be an educational resource. Then, the $k$ dimensions of the opportunity set $\mathbf{c}_{i}^{m}(x) \subseteq \mathbb{R}_{+}^{k}$ represent the types of skills, and each element $\mathbf{b}_{i}=\left(b_{i f}\right)_{f \in\{1, \ldots, k\}} \in \mathbf{c}_{i}^{m}(x)$ implies that individual $i$ can develop the level of each skill $f$ up to $b_{i f}$, whenever he is educated with the educational support $x$ and some amount of his own effort. The difference of native talents among individuals is reflected in the difference of opportunity correspondences among them. In this setting, the bargaining problem would be to assign opportunities for future skills by allocating educational resources.

Example 2: The WTO consists of many member countries and a part of its functions is to settle disputes among its member countries. Disputes between or among member countries are really about net trades of goods, services or capital. The Dispute Settlement Body of the WTO is thus to make recommendations as how to structure net trades among the affected member countries. ${ }^{3}$ Each member country is concerned about, for example, the aggregagte employment rate, the growth rates of several sectors like manufacturing, agriculture, and service, and the health condition of its population. These concerns of a member country correspond to our notion of achievements. Each member country's interests can be captured by the country's opportunity sets representing opportunities to have a certain degree of employment rate, to have reasonable growth rates for its concerned sectors, and to offer its population a good health. The bargaining problem can then be interpreted as follows. The Dispute Settlement Body in the WTO mechanism acts as the fair arbitrator and it recommends the settlements that affect net trade based on equal opportunities for the disputed member countries along the factors that we discussed above.

Example 3: Our last example concerns the allocation of the budget by a central government to its several local jurisdictions. In many cases, the

\footnotetext{
${ }^{3}$ Quite often, disputes seemingly are about things like access to member countries' markets and information, legal protection concerning trades from member countries, or pricing rules. These are rules governing trade between and among nations and they have direct effect on net trade between member countries. As a consequence, we can interpret that disputes are really about net trade.
} 
allocation of the budget by the central government to its local jurisdictions intends for different localities to have equal opportunities for growth and for access to clean water, for example. Growth and access to clean water are two of the many factors that different local jurisdictions are concerned about, and local governments are concerned about their opportunities along these factors. The bargaining problem in this example can thus be viewed as the fair arbitrator, the central government, makes budgetary allocations on the basis of equal opportunies for different local jurisdictions along those factors such as growth and environmenal quality of each region.

\section{Three Bargaining Solutions}

In this section, we will introduce three bargaining solutions in our context. To do this, we need an objective measure to evaluate the relative wellness of alternative opportunity sets. All of the three solutions that we will introduce in the next subsection are based on the type of such objective measure. With different objective measures, the recommendation by a bargaining solution can be different. This is because there is no a priori, unique measure for evaluating different opportunity sets appropriately. Note that, in the case of the standard bargaining models with individual utilities, there is no complexity in measuring individuals' utilities. In our context, however, we need to start with the discussion on how to measure opportunity sets.

We therefore begin by defining a social ordering over opportunity sets. Such an ordering gives us information about objective measures for evaluating various opportunity sets.

\subsection{Properties on orderings over opportunity sets}

An objective measure of alternative opportunity sets is formalized as a binary relation $R \subseteq \mathcal{K} \times \mathcal{K}$. The relation $R$ satisfies reflexivity: [for all $C \in \mathcal{K}$, $(C, C) \in R$ ], completeness: [for all $C, C^{\prime} \in \mathcal{K},\left(C, C^{\prime}\right) \in R$ or $\left(C^{\prime}, C\right) \in R$ ], and transitivity: [for all $C, C^{\prime}, C^{\prime \prime} \in \mathcal{K}$, if $\left(C, C^{\prime}\right) \in R \&\left(C^{\prime}, C^{\prime \prime}\right) \in R$, then $\left(C, C^{\prime \prime}\right) \in R$ ]. Thus, $R$ is an ordering over $\mathcal{K}$. Note $P$ and $I$ are respectively the asymmetric and symmetric parts of $R$.

For all $C, C^{\prime} \in \mathcal{K}$, we write $C>C^{\prime}$ if for all $\mathbf{b}^{\prime} \in C^{\prime}$, there exists $\mathbf{b} \in C$ such that $\mathbf{b} \gg \mathbf{b}^{\prime}$. Note that, given the comprehensiveness of opportunity sets in $\mathcal{K}$, when $C>C^{\prime}$, necessarily, we have $C^{\prime}$ as a proper subset of $C$. 
In this paper, we assume that the ordering $R$ on $\mathcal{K}$ satisfies the following two properties:

Monotonicity: For all $C, C^{\prime} \in \mathcal{K}$, if $C \supseteq C^{\prime}$ then $\left(C, C^{\prime}\right) \in R$, and if $C>C^{\prime}$, then $\left(C, C^{\prime}\right) \in P$.

Cardinal Representability: There exists a real-valued, continuous function $G: \mathcal{K} \rightarrow \mathbb{R}_{+}$such that for all $C, C^{\prime} \in \mathcal{K}$,

$$
G(C) \geq G\left(C^{\prime}\right) \Leftrightarrow\left(C, C^{\prime}\right) \in R,
$$

where $G$ is unique up to a positive affine transformation.

It may be noted that, in our context, Monotonicity is a fairly noncontrovercial property and it essentially requires that a "bigger" opportunity set be ranked higher than a "smaller" opportunity set. Similar conditions have been used in the literature on ranking opportunity sets, see for example, Gaertner and Xu (2006), Pattanaik and Xu (2006), and Xu (2002, 2004). Cardinal Representability requires the function $G$ which represents the ordering be cardinal. See Pattanaik and Xu (2000), Xu (2004), and Savaglio and Vannucci (2006) for examples of such cardinal representations of a binary relation over opportunity sets.

In addition to the above two basic properties to be imposed on the ordering $R$, in some cases, we also impose the following property on the ordering $R$ :

Homotheticity: For all $C, C^{\prime} \in \mathcal{K}$, and all $\alpha>0,\left(C, C^{\prime}\right) \in R \Leftrightarrow\left(\alpha \cdot C, \alpha \cdot C^{\prime}\right) \in$ $R$.

In our context of bargaining over opportunity assignments, homotheticity of $G$ may be viewed as a reasonable property. Recall that, in our setting, the opportunity set $\{\mathbf{0}\}$ can be regarded as the "disagreement point". Then, for any opportunity set $C$, there is a chance that an individual has the opportunity set $C$ when an agreement is reached (the individual would end up with $\{\mathbf{0}\}$ when there is no agreement reached). Let $p>0$ be the probability that an agreement can be reached. Then, homotheticity of $G$ basically says that the ranking of any two opportunity sets $C$ and $C^{\prime}$ should be independent of this probability $p$ so that $\left(C, C^{\prime}\right) \in R$ iff $\left(p \cdot C+(1-p) \cdot\{\mathbf{0}\}, p \cdot C^{\prime}+(1-p) \cdot\{\mathbf{0}\}\right) \in R$; that is, $\left(C, C^{\prime}\right) \in R$ iff $\left(p \cdot C, p \cdot C^{\prime}\right) \in R$. 


\subsection{Three solutions}

Given a social evaluation of opportunity sets $R$ satisfying Monotonicity and Cardinal Representability and its representation $G$, the three solutions we consider in the paper are defined as follows:

Nash Solution: A bargaining solution $F^{N A}$ is the Nash solution if, for every $\mathcal{C} \in \Sigma$,

$F^{N A}(\mathcal{C})=\left\{\mathbf{C} \in \mathcal{C} \mid \prod_{i \in N}\left(G\left(C_{i}\right)-G(\{\mathbf{0}\})\right) \geq \prod_{i \in N}\left(G\left(C_{i}^{\prime}\right)-G(\{\mathbf{0}\})\right)\left(\forall \mathbf{C}^{\prime} \in \mathcal{C}\right)\right\}$.

Egalitarian Solution: A bargaining solution $F^{E}$ is the egalitarian solution if, for every $\mathcal{C} \in \Sigma, \mathbf{C} \in F^{E}(\mathcal{C})$ implies that: (1) there is no other $\mathbf{C}^{\prime} \in \mathcal{C}$ such that $\left(C_{i}^{\prime}, C_{i}\right) \in P$ for all $i \in N$; and (2) $\left(C_{i}, C_{j}\right) \in I$ holds for any $i, j \in N$.

Kalai-Smorodinsky Solution: A bargaining solution $F^{K S}$ is the KalaiSmorodinsky solution if, for every $\mathcal{C} \in \Sigma, \mathbf{C} \in F^{K S}(\mathcal{C})$ implies that: (1) there is no other $\mathbf{C}^{\prime} \in \mathcal{C}$ such that $\left(C_{i}^{\prime}, C_{i}\right) \in P$ for all $i \in N$; and (2) $\frac{\left.G\left(C_{i}\right)-G(\{\mathbf{0}\})\right)}{\left(G\left(m_{i}(\mathcal{C})\right)-G(\{\mathbf{0}\})\right.}=\frac{G\left(C_{j}\right)-G(\{\mathbf{0}\})}{G\left(m_{j}(\mathcal{C})\right)-G(\{\mathbf{0}\})}$.

\section{Axioms on bargaining solutions}

In this section, we shall present and discuss axioms on bargaining solutions over opportunity assignments. The first two axioms are the corresponding efficiency type axioms in standard bargaining models.

Efficiency (E): For each $\mathcal{C} \in \Sigma$ and each $\mathbf{C}=\left(C_{i}\right)_{i \in N} \in F(\mathcal{C})$, there is no $\mathbf{C}^{\prime}=\left(C_{i}^{\prime}\right)_{i \in N} \in \mathcal{C}$ such that for every $i \in N, C_{i}^{\prime} \supseteq C_{i}$, and for some $j \in N$, $C_{j}^{\prime}>C_{j}$.

Weak Efficiency (WE): For each $\mathcal{C} \in \Sigma$ and each $\mathbf{C}=\left(C_{i}\right)_{i \in N} \in F(\mathcal{C})$, there is no $\mathbf{C}^{\prime}=\left(C_{i}^{\prime}\right)_{i \in N} \in \mathcal{C}$ such that for every $i \in N, C_{i}^{\prime}>C_{i}$.

Therefore, the axiom (E) requires that, for a opportunity assignment $\mathbf{C}$ to be considered as a part of the solution to a problem, there must not exist another feasible opportunity assignment $\mathbf{C}^{\prime}$ such that $C_{i} \subseteq C_{i}^{\prime}$ for all $i \in N$ and 
$C_{i}^{\prime}>C_{i}$ for at least one $i \in N$. (E) corresponds to the usual efficiency axiom in the standard bargaining model. (WE) is a weaker requirement than (E) and requires that the solution should not select an opportunity assignment that is strictly dominated by another feasible opportunity assingment.

To introduce our next two axioms, we first define notions of a symmetric opportunity assignment and related concepts. Let $\mathcal{C} \in \Sigma$. We say that $\mathcal{C} \in \Sigma$ is symmetric if for every permutation $\pi: N \rightarrow N$, and for every $\mathbf{C}=\left(C_{i}\right)_{i \in N} \in \mathcal{C}, \pi(\mathbf{C}) \equiv\left(C_{\pi(i)}\right)_{i \in N} \in \mathcal{C}$ holds. If $\mathbf{C}^{*}=\left(C_{i}^{*}\right)_{i \in N} \in \mathcal{K}^{n}$ is such that $C_{i}^{*}=C_{j}^{*}$ for any $i, j \in N$, then $\mathbf{C}^{*}$ is said to be a symmetric opportunity assignment.

Let $\Lambda \subseteq \mathbb{R}_{+}$be a set of index numbers with the minimal value $0 \in \Lambda$. Let $\left\{\mathbf{C}^{s}\right\}_{s \in \Lambda} \subseteq \mathcal{K}^{n}$ be a sequence of symmetric assignments such that (a) for any $s \in \Lambda, \mathbf{C}^{s} \in \mathcal{K}^{n}$ is a symmetric assignment; (b) for any $s, s^{\prime} \in \Lambda$ with $s<s^{\prime}$, $C^{s} \subseteq C^{s^{\prime}}$; and (c) $\mathbf{C}^{0}=\{\mathbf{0}\}$. We call such $\left\{\mathbf{C}^{s}\right\}_{s \in \Lambda}$ a monotone path of symmetric assignments. In general, $\Lambda$ is not a closed set. If $\Lambda$ is closed, then there exists the maximal symmetric assignment $\mathbf{C}^{s^{*}}$ in $\left\{\mathbf{C}^{s}\right\}_{s \in \Lambda}$. In this case, $\left\{\mathbf{C}^{s}\right\}_{s \in \Lambda}$ is called a closed monotone path of symmetric assignments. $\mathcal{C} \in \Sigma$ is said to be symmetric with a closed monotone symmetric path of symmetric assignments $\left\{\mathbf{C}^{s}\right\}_{s \in \Lambda}$ if $\mathcal{C}$ is symmetric, and $\left\{\mathbf{C}^{s}\right\}_{s \in \Lambda} \subseteq \mathcal{C}$ is such that the maximal symmetric assignment $\mathbf{C}^{s^{*}}$ is a weakly efficient assignment in $\mathcal{C}$.

Anonymity (A): For each $\mathcal{C} \in \Sigma$, if $\mathcal{C}$ is symmetric, then for each $\mathbf{C} \in$ $F(\mathcal{C})$ and each permutation $\pi$ over $N, \pi(\mathbf{C}) \in F(\mathcal{C})$.

Symmetry (S): For each $\mathcal{C} \in \Sigma$, if $\mathcal{C}$ is symmetric with a closed monotone symmetric path of symmetric assignments $\left\{\mathbf{C}^{s}\right\}_{s \in \Lambda}$, then $F(\mathcal{C}) \cap\left\{\mathbf{C}^{s}\right\}_{s \in \Lambda} \neq$ $\varnothing$, and for no $\mathbf{C} \in F(\mathcal{C}), C_{i}>C_{j}$ for some $i, j \in N$.

The axiom (A) requires that, for each symmetric problem, if an opportunity assignment $\mathbf{C}$ is selected by the solution, so is every permutation of C. The axiom (S) stipulates that, for each symmetric problem with a closed monotone symmetric path of symmetric assignments $\left\{\mathbf{C}^{s}\right\}_{s \in \Lambda}$, then the solution selects at least one opportunity assignment from $\left\{\mathbf{C}^{s}\right\}_{s \in \Lambda}$, and further, for no opportunity assignment selected by the solution is such that one individual's opportunity set strictly dominates another individual's opportunity set.

The following axioms are analogous to the axioms of contraction independence and of weak contraction independence in standard bargaining models: 
Contraction Independence $(\mathbf{C I}):$ For each $\mathcal{C}, \mathcal{C}^{\prime} \in \Sigma$ with $\mathcal{C} \supseteq \mathcal{C}^{\prime}$, if $F(\mathcal{C}) \cap \mathcal{C}^{\prime} \neq \varnothing$, then $F\left(\mathcal{C}^{\prime}\right)=F(\mathcal{C}) \cap \mathcal{C}^{\prime}$

Weak Contraction Independence (WCI): For each $\mathcal{C}, \mathcal{C}^{\prime} \in \Sigma$ with $\mathcal{C} \supseteq$ $\mathcal{C}^{\prime}$ and $m_{i}(\mathcal{C})=m_{i}\left(\mathcal{C}^{\prime}\right)$ for all $i \in N$, if $F(\mathcal{C}) \cap \mathcal{C}^{\prime} \neq \varnothing$, then $F\left(\mathcal{C}^{\prime}\right)=$ $F(\mathcal{C}) \cap \mathcal{C}^{\prime}$

The axiom (CI) corresponds to Nash's Independence of Irrelevant Alternatives in standard bargaining models. It requires that if an opportunity assignment is chosen from a "larger" problem and is still available when the larger problem shrinks to a smaller problem, then it should be chosen from the smaller problem as well. (WCI) is a restrictive version of (CI) by requiring two problems of having the same "ideal" opportunity assignment. Its origin in standard bargaining models goes back to Yu (1973) (see Xu and Yoshihara (2006) for an application of this axiom in characterizing the Kalai-Smorodinsky solution for nonconvex problems).

The next axiom is perhaps most controversial in our context:

Invariance to Affine Changes in opportunity Correspondences (IACC): For each $\mathcal{C}, \mathcal{C}^{\prime} \in \Sigma$, if there exists a vector $\mathbf{a}=\left(a_{i}\right)_{i \in N} \in \mathbb{R}_{++}^{n}$ such that $\mathcal{C}^{\prime}=\left\{\mathbf{a} \cdot \mathbf{C}=\left(a_{i} \cdot C_{i}\right)_{i \in N} \subseteq \mathbb{R}_{+}^{k n} \mid \mathbf{C} \in \mathcal{C}\right\}$, then $\mathbf{C} \in F(\mathcal{C}) \Leftrightarrow \mathbf{a} \cdot \mathbf{C} \in F\left(\mathcal{C}^{\prime}\right)$.

We first note that the axiom of (IACC) cannot be interpreted as an analogy to the scale invariance axiom of standard bargaining problems over utility allocations. The $k$ components of living conditions are assumed to be common to all individuals and are supposed to be objectively measurable. These units are used to identify each individual's attainable living-condition vectors and each individual's opportunity sets. Consequently, the fair arbitrator can make interpersonal comparisons of living-condition vectors and interpersonal comparisons of opportunity sets. The common social ordering $R$ over opportunity sets for all individuals can be justified on the ground that there exist such $k$ units for measuring living-condition levels.

Given this implicit presumption in our framework, we cannot interpret the change of one individual's opportunity set from $C_{i}$ to $C_{i}^{\prime}=a_{i} \cdot C_{i}$ as the change in units of $k$ living conditions for measuring individual $i$ 's wellbeing. If it was so, other individuals' opportunity sets would have also been transformed by the same affine parameter $a_{i}$. The axiom (IACC), however, permits the situation of $a_{i} \neq a_{j}$ between two different individuals $i$ and $j$. 
Therefore, we are reluctant to interpret the axiom (IACC) in terms of the invariance to interpersonally different units of $k$ living conditions.

The most plausible interpretation of the change from $C_{i}$ to $C_{i}^{\prime}=a_{i} \cdot C_{i}$ is, we think, the change of $i$ 's opportunity correspondence. In other words, individual $i$ 's "productivity" of living conditions is either improved $\left(a_{i}>1\right)$ or deteriorated $\left(a_{i}<1\right)$. The axiom (IACC) then suggests that the change in relative bargaining powers as reflected in each individual $i$ 's $a_{i}$ should not affect the underlying resource allocations of commodities. In a sense, the axiom seems to protect a relatively weak bargainer from a relatively strong bargainer. On the other hand, the axiom (IACC) also suggests that the society should not compensate someone for the deterioration of his "productivity" of living conditions through a redistributive policy.

Our final axiom is an informational requirement on a solution to a problem and is stated below:

Informational Invariance (II) : For each $\mathcal{C} \in \Sigma$ and each $\mathbf{C}=\left(C_{i}\right)_{i \in N}, \mathbf{C}^{\prime}=$ $\left(C_{i}^{\prime}\right)_{i \in N} \in \mathcal{C}$, if $\mathbf{C} \in F(\mathcal{C})$ and $\left(C_{i}^{\prime}, C_{i}\right) \in I$ for all $i \in N$, then $\mathbf{C}^{\prime} \in F(\mathcal{C})$.

According to the axiom (II), if two opportunity assignments are "equivalent" in the sense that the two opportunity sets for each and every individual specified by the corresponding opportunity assignments are ranked equally, then whenever one opportunity assignment is chosen by the solution, the other opportunity assignment should be chosen by the solution as well. The axiom (II) thus implies that the informational requirement in our context is contained exclusively in the social evaluation ordering $R$. A similar axiom, called No Discrimination, is discussed by Thomson (1983) in the context of fair allocation problems.

\section{Main Results and Interpretations}

Before we present our characterization results, the following observations are useful throughout this section. Let $G$ be the real-valued, cardinal representation of the social ordering $R$. For each bargaining problem $\mathcal{C} \in \Sigma$, we define

$$
G(\mathcal{C}) \equiv\left\{G(\mathbf{C})=\left(G\left(C_{i}\right)\right)_{i \in N} \in \mathbb{R}_{+}^{n} \mid \mathbf{C} \in \mathcal{C}\right\} .
$$

Let $\partial G(\mathcal{C})$ be the upper boundary of $G(\mathcal{C})$. Since $\mathcal{C}$ is derived from an underlying economic environment $\mathbf{e}=\left(M, \mathbf{c}^{m}, \bar{x}\right) \in \mathcal{E}$, where $\mathbf{c}^{m}$ is a profile 
of opportunity correspondences satisfying the requirements (a), (b), (c), and $(\mathrm{d})$, and $G$ is continuous on $\mathcal{K}, \partial G(\mathcal{C})$ constitutes a connected set in $\mathbb{R}_{+}^{n}$. Moreover, since $\mathcal{C}$ is comprehensive 4 by the requirements (a), (b), and (d) of opportunity correspondences, $G(\mathcal{C})$ must be comprehensive. Finally, by choosing $G(\{\mathbf{0}\})=0$ for the zero vector $\mathbf{0} \in \mathbb{R}_{+}^{k}, G(\mathcal{C})$ has $\mathbf{0} \in \mathbb{R}_{+}^{n}$ as its element, since $\underbrace{(\{\mathbf{0}\}, \ldots,\{\mathbf{0}\})}_{n \text {-times }} \in \mathcal{C}$.

\subsection{The egalitarian solution}

We first give a characterization of the egalitarian solution and its proof follows.

Theorem 1: The egalitarian solution $F^{E}$ is the unique solution satisfying (WE), (S), (CI) and (II).

Proof. First, it may be checked that $F^{E}$ satisfies the four axioms of the theorem.

Next, we show that if a solution $F$ satisfies (WE), (S), (CI) and (II), then $F=F^{E}$. Let us choose any bargaining problem $\mathcal{C} \in \Sigma$, which is derived from an underlying economic environment $\mathbf{e}=\left(M, \mathbf{c}^{m}, \bar{x}\right) \in \mathcal{E}$. Suppose $F \neq F^{E}$. By $(\mathrm{II})$, we can suppose that $G(F(\mathcal{C})) \neq G\left(F^{E}(\mathcal{C})\right)$ for this $\mathcal{C} \in \Sigma$. Then, there exists $\overline{\mathbf{G}}^{*} \in G(F(\mathcal{C})) \backslash G\left(F^{E}(\mathcal{C})\right)$. Given $\mathcal{C}$, we define $\mathcal{C}(i) \equiv\left\{C_{i}^{\prime} \in \mathcal{K} \mid \exists \mathbf{C}_{-i} \in \mathcal{K}^{n-1}:\left(C_{i}^{\prime}, \mathbf{C}_{-i}\right) \in \mathcal{C}\right\}$ for each $i \in N$. For each $i \in N$ and each $r \in \mathbb{R}_{+}$, define

$$
\mathcal{C}(i ; r) \equiv\left\{C_{i}^{\prime} \in \mathcal{C}(i) \mid G\left(C_{i}^{\prime}\right)=r\right\} .
$$

Let $\mathbf{r}^{*} \equiv \overline{\mathbf{G}}^{*}$. Consider $\operatorname{comp}\left\{\mathbf{r}^{*}\right\} \equiv\left\{\mathbf{r} \in \mathbb{R}_{+}^{n}: \mathbf{r} \leq \mathbf{r}^{*}\right\}$, and $\mathcal{C}^{*} \equiv$ $G^{-1}\left(\operatorname{comp}\left\{\mathbf{r}^{*}\right\}\right)$.

Insert Figure 1 around here.

Since $G$ is continuous, we can choose a continuous sequence $\mathcal{C}_{s}^{*} \subseteq \mathcal{C}^{*}$ in which (i) for each $\mathbf{r} \in \operatorname{comp}\left\{\mathbf{r}^{*}\right\}$, there exists a unique $\left(C_{i}^{r_{i}}\right)_{i \in N} \in \mathcal{C}_{s}^{*}$ such that $C_{i}^{r_{i}} \in \mathcal{C}\left(i ; r_{i}\right)$ for each $i \in N$; (ii) for each $\mathbf{r}, \mathbf{r}^{\prime} \in \operatorname{comp}\left\{\mathbf{r}^{*}\right\}$, for each $i \in N, C_{i}^{r_{i}}=C_{i}^{r_{i}^{\prime}}$ holds if $r_{i}=r_{i}^{\prime}$ and $C_{i}^{r_{i}}>C_{i}^{r_{i}^{\prime}}$ if $r_{i}>r_{i}^{\prime}$; and (iii)

\footnotetext{
${ }^{4} \mathcal{C}$ is comprehensive if, for each $\mathbf{C} \in \mathcal{C}$ and each $i \in N$ with $C_{i} \neq\{\mathbf{0}\}$, there exists $\mathbf{C}^{\prime}$ $\in \mathcal{C}$ such that $C_{i}^{\prime}<C_{i}$ and $C_{j}^{\prime} \subseteq C_{j}$ for all $j \in N \backslash\{i\}$.
} 
for any $\mathbf{r}^{\prime} \in \operatorname{comp}\left\{\mathbf{r}^{*}\right\}, \lim _{\mathbf{r} \rightarrow \mathbf{r}^{\prime}}\left(C_{i}^{r_{i}}\right)_{i \in N}=\left(C_{i}^{r_{i}^{\prime}}\right)_{i \in N}$ holds. By definition, $G\left(\mathcal{C}_{s}^{*}\right)=\operatorname{comp}\left\{\mathbf{r}^{*}\right\}$.

Now, by using the information of $\mathcal{C}_{s}^{*}$, we are ready to construct $\mathbf{e}^{*}=$ $\left(M^{*}, \widehat{\mathbf{c}}^{1}, \bar{x}^{*}\right) \in \mathcal{E}$, in which $M^{*} \cap M=\varnothing, \# M^{*}=1$, and $\bar{x}^{*}=1$. Moreover, for each $i \in N$, the opportunity correspondence $\widehat{c}_{i}^{1}$ is defined as follows:

(i) for all $x \in\left[0, \frac{1}{n}\right], \widehat{c}_{i}^{1}(x)=C_{i}^{r_{i}^{\prime}}$ with $r_{i}^{\prime}=n x \cdot r_{i}^{*}$; and

(ii) for all $x \in\left(\frac{1}{n}, 1\right], \widehat{c}^{1}(x)=C_{i}^{r_{i}^{*}}$.

Then, consider $\mathcal{C}^{* *} \equiv \mathcal{C}\left(\mathbf{e}^{*}\right) \in \Sigma$.

By $(\mathrm{CI}), \mathbf{r}^{*} \in G\left(F\left(\mathcal{C}^{* *}\right)\right)$. Next, consider $\mathcal{C}^{\triangle} \equiv \cup_{\pi \in \Pi} \pi\left(\mathcal{C}^{* *}\right)$.

Insert Figure 2 around here.

We will construct a new economy $\mathbf{e}^{\triangle}=\left(M^{\triangle}, \mathbf{c}^{\triangle n}, \bar{x}^{\triangle}\right) \in \mathcal{E}$ such that $\mathcal{C}\left(\mathbf{e}^{\triangle}\right) \supseteq \mathcal{C}^{\triangle}$ and $G\left(\mathcal{C}\left(\mathbf{e}^{\triangle}\right)\right)=G\left(\mathcal{C}^{\triangle}\right)$. First, define an opportunity correspondence $c^{n}:\left[0, \bar{x}^{*}\right]^{n} \rightarrow \mathbb{R}_{+}^{k}$ by: for every $x=\left(x_{i}\right)_{i \in N} \in\left[0, \bar{x}^{*}\right]^{n}$, $c^{n}(x) \equiv \cup_{i \in N} \widehat{c}_{i}^{1}\left(x_{i}\right)$. Consider $\mathbf{e}^{(n)}=\left(M^{*(n)}, \mathbf{c}^{n},\left(\bar{x}^{*}\right)^{n}\right) \in \mathcal{E}$, where $c_{i}^{n}=$ $c^{n}$ for every $i \in N$, and $\left(\bar{x}^{*}\right)^{n} \equiv \underbrace{\left(\bar{x}^{*}, \ldots, \bar{x}^{*}\right)}_{n \text {-times }}$. Then, $\mathcal{C}\left(\mathbf{e}^{(n)}\right) \supseteq \mathcal{C}^{\triangle}$, but $G\left(\mathcal{C}\left(\mathbf{e}^{(n)}\right)\right) \supsetneq G\left(\mathcal{C}^{\triangle}\right)$.

Insert Figure 3 around here.

Note that both $G\left(\mathcal{C}\left(\mathbf{e}^{(n)}\right)\right)$ and $G\left(\mathcal{C}^{\triangle}\right)$ are symmetric, and $\partial G\left(\mathcal{C}\left(\mathbf{e}^{(n)}\right)\right)$ is homeomorphic to $\partial G\left(\mathcal{C}^{\triangle}\right)$. Thus, we can take a continuous bijection $\boldsymbol{\lambda}: \partial G\left(\mathcal{C}\left(\mathbf{e}^{(n)}\right)\right) \rightarrow \partial G\left(\mathcal{C}^{\triangle}\right)$ such that for any $\mathbf{r} \in \partial G\left(\mathcal{C}\left(\mathbf{e}^{(n)}\right)\right)$, there exists a scalor $\lambda^{\mathbf{r}}>0$ such that $\boldsymbol{\lambda}(\mathbf{r})=\left(\lambda^{\mathbf{r}} \cdot r_{i}\right)_{i \in N} \in \partial G\left(\mathcal{C}^{\triangle}\right)$. Define an opportunity correspondence $c^{\triangle n}$ as follows: For any $\left(\lambda^{\mathbf{r}} \cdot r_{i}\right)_{i \in N} \in$ $\partial G\left(\mathcal{C}^{\triangle}\right)$, any $i \in N$, any $r \in\left[0, \lambda^{\mathbf{r}} \cdot r_{i}\right]$, and any $x \in\left(c^{n}\right)^{-1} \circ G^{-1}(r)$, let $c^{\triangle n}(x)=c^{n}(x)$; and for any $\mathbf{r} \in \partial G\left(\mathcal{C}\left(\mathbf{e}^{(n)}\right)\right)$, if $\lambda^{\mathbf{r}} \cdot r_{i}<r_{i}$, then for any $r \in\left(\lambda^{\mathbf{r}} \cdot r_{i}, r_{i}\right]$, and any $x \in\left(c^{n}\right)^{-1} \circ G^{-1}(r)$, let $c^{\triangle n}(x) \in G^{-1}\left(\lambda^{\mathbf{r}} \cdot r_{i}\right)$. Then, $\mathbf{e}^{\triangle} \equiv\left(M^{*(n)}, \mathbf{c}^{\triangle n},\left(\bar{x}^{*}\right)^{n}\right) \in \mathcal{E}$, where $\mathbf{c}^{\triangle n}=\underbrace{\left(c^{\Delta n}, \ldots, c^{\triangle n}\right)}_{n \text {-times }}$. By definition, $\mathcal{C}\left(\mathbf{e}^{\triangle}\right) \supseteq \mathcal{C}^{\triangle}$, and $G\left(\mathcal{C}\left(\mathbf{e}^{\triangle}\right)\right)=G\left(\mathcal{C}^{\triangle}\right)$.

From the construction of $\mathcal{C}\left(\mathbf{e}^{\triangle}\right)$, by (WE), (S), and (II), we must have the following:

$$
G\left(F\left(\mathcal{C}\left(\mathbf{e}^{\triangle}\right)\right)\right)=\left\{G\left(\mathbf{c}^{\triangle n}\left(\left(\frac{1^{*}}{n}\right)^{n}\right)\right)\right\},
$$




$$
\begin{aligned}
& \text { where } \mathbf{c}^{\triangle n}\left(\left(\frac{1^{*}}{n}\right)^{n}\right)=\underbrace{\left(c^{\triangle n}\left(\left(\frac{1^{*}}{n}\right)^{n}\right), \ldots, c^{\triangle n}\left(\left(\frac{1^{*}}{n}\right)^{n}\right)\right)}_{n \text {-times }} \\
& \quad \text { and }\left(\frac{1^{*}}{n}\right)^{n}=\underbrace{\left(\frac{1^{*}}{n}, \ldots, \frac{1^{*}}{n}\right)}_{n \text {-times }} .
\end{aligned}
$$

Let $\mathbf{r}^{\triangle E} \equiv G\left(\mathbf{c}^{\triangle n}\left(\left(\frac{1^{*}}{n}\right)^{n}\right)\right)$, which is the egalitarian outcome for the problem $\mathcal{C}\left(\mathbf{e}^{\triangle}\right)$.

Insert Figure 4 around here.

Since $\mathbf{r}^{\triangle E} \in \partial G\left(\mathcal{C}^{* *}\right)$, there exists $\mathbf{C}^{\mathbf{r}^{\triangle E}} \in \mathcal{C}^{* *}$ such that $G\left(\mathbf{C}^{\mathbf{r}^{\triangle E}}\right)=\mathbf{r}^{\triangle E}$. Thus, by $(\mathrm{CI}),\left\{\mathbf{r}^{\triangle E}\right\}=G\left(F\left(\mathcal{C}^{* *}\right)\right)$, which is a desired contradiction, since $\mathbf{r}^{*} \in G\left(F\left(\mathcal{C}^{* *}\right)\right)$.

Insert Figure 5 around here.

Thus, $G(F(\mathcal{C})) \backslash G\left(F^{E}(\mathcal{C})\right)=\varnothing$. By (II), clearly, $F=F^{E}$.

\subsection{The Kalai-Smorodinsky solution}

We now turn to a characterization of the Kalai-Smorodinsky solution.

Theorem 2: Let the social evaluation of opportunity sets $R$ be homothetic. Then, the Kalai-Smorodinsky solution $F^{K}$ is the unique solution satisfying (WE), (S), (IACC), (WCI) and (II).

Proof. First, it can be checked that $F^{K}$ satisfies the five axioms of the theorem. Next, we show that if a solution $F$ satisfies the axioms of (WE), (S), (IACC), (WCI) and (II), then $F=F^{K}$. Let $G: \mathcal{K} \rightarrow \mathbb{R}_{+}$cardinally represent the homothetic ordering $R$ so that $G$ is homogeneous of degree $l$ for some positive integer $l$. Let $\mathcal{C} \in \Sigma$ be an arbitrary problem derived from an underlying economic environment $\mathbf{e}=\left(M, \mathbf{c}^{m}, \bar{x}\right) \in \mathcal{E}$. Given this $G(\mathcal{C})$, for each $i \in N$, let $m_{i}(G(\mathcal{C}))$ be the point such that $\left(m_{i}(G(\mathcal{C})), \mathbf{0}_{-i}\right) \in G(\mathcal{C})$, and $m_{i}(G(\mathcal{C})) \geq \bar{G}_{i}$ for any $\overline{\mathbf{G}} \in G(\mathcal{C})$ with $\bar{G}_{i}$ as its $i$-th component. Also, let $G\left(F^{K}(\mathcal{C})\right) \in \partial G(\mathcal{C})$ be the point in the $G$-space of the KalaiSmorodinsky solution. Now, let us choose the $n$ dimensional positive vector $\mathbf{a}=\left(a_{i}\right)_{i \in N} \in \mathbb{R}_{++}^{n}$ appropriately, so that

$$
\mathcal{C}^{\prime}=\mathbf{a} \cdot \mathcal{C} \equiv\left\{\mathbf{a} \cdot \mathbf{C}=\left(a_{i} \cdot C_{i}\right)_{i \in N} \subseteq \mathbb{R}_{+}^{k n} \mid \mathbf{C} \in \mathcal{C}\right\}
$$


has the property that, for each $i \in N, m_{i}\left(G\left(\mathcal{C}^{\prime}\right)\right)=1$. Note that

$$
\begin{aligned}
G\left(F^{K}\left(\mathcal{C}^{\prime}\right)\right) & =\mathbf{a}^{l} \cdot G\left(F^{K}(\mathcal{C})\right) \equiv \overline{\mathbf{G}}^{K\left(\mathcal{C}^{\prime}\right)}, \\
\text { where } \mathbf{a}^{l} & \equiv\left(a_{i}^{l}\right)_{i \in N} \text { and } a_{i}^{l} \equiv \underbrace{a_{i} \cdot a_{i} \cdot \ldots \cdot a_{i}}_{l \text {-times }}
\end{aligned}
$$

has the property that, for each $i \in N, \bar{G}_{i}^{K\left(\mathcal{C}^{\prime}\right)}=r^{K} \in[0,1]$.

Suppose $F \neq F^{K}$. By (II), we can suppose that $G(F(\mathcal{C})) \neq G\left(F^{K}(\mathcal{C})\right)$ for this $\mathcal{C} \in \Sigma$. Then, there exists $\overline{\mathbf{G}}^{*} \in G(F(\mathcal{C})) \backslash G\left(F^{K}(\mathcal{C})\right)$. By (IACC), $\mathbf{a}^{l} \cdot \overline{\mathbf{G}}^{*} \in G\left(F\left(\mathcal{C}^{\prime}\right)\right) \backslash G\left(F^{K}\left(\mathcal{C}^{\prime}\right)\right)$. Let $\mathbf{r}^{*} \equiv \mathbf{a}^{l} \cdot \overline{\mathbf{G}}^{*}$. Given the bargaining problem $\mathcal{C}^{\prime}$, we define

$$
\mathcal{C}^{\prime}(i) \equiv\left\{C_{i}^{\prime} \in \mathcal{K} \mid \exists \mathbf{C}_{-i} \in \mathcal{K}^{n-1}:\left(C_{i}^{\prime}, \mathbf{C}_{-i}\right) \in \mathcal{C}^{\prime}\right\} .
$$

Next, for each $i \in N$ and each $r \in \mathbb{R}_{+}$, we define

$$
\mathcal{C}^{\prime}(i ; r) \equiv\left\{C_{i}^{\prime} \in \mathcal{C}^{\prime}(i) \mid G\left(C_{i}^{\prime}\right)=r\right\}
$$

Let $\mathbf{e}^{i} \equiv\left(m_{i}\left(G\left(\mathcal{C}^{\prime}\right)\right), \mathbf{0}_{-i}\right)$ for each and every $i \in N$.

Consider comp $\left\{\mathbf{r}^{*}, \mathbf{e}^{1}, \ldots, \mathbf{e}^{n}\right\} \equiv\left\{\mathbf{r} \in \mathbb{R}_{+}^{n}: \mathbf{x} \leq \mathbf{r}^{*}\right\} \cup\left\{\mathbf{r} \in \mathbb{R}_{+}^{n}: \mathbf{r} \leq\right.$ $\left.\mathbf{e}^{1}\right\} \cup \ldots \cup\left\{\mathbf{r} \in \mathbb{R}_{+}^{n}: \mathbf{r} \leq \mathbf{e}^{n}\right\}$, and $\mathcal{C}^{\prime \prime} \equiv G^{-1}\left(\operatorname{comp}\left\{\mathbf{r}^{*}, \mathbf{e}^{1}, \ldots, \mathbf{e}^{n}\right\}\right)$. Since $G$ is continuous, we can choose a continuous sequence $\mathcal{C}_{s}^{\prime \prime} \subseteq \mathcal{C}^{\prime \prime}$ in which (i) for each $\mathbf{r} \in \operatorname{comp}\left\{\mathbf{r}^{*}, \mathbf{e}^{1}, \ldots, \mathbf{e}^{n}\right\}$, there exists a unique $\left(C_{i}^{r_{i}}\right)_{i \in N} \in \mathcal{C}_{s}^{\prime \prime}$ such that $C_{i}^{r_{i}} \in \mathcal{C}^{\prime}\left(i ; r_{i}\right)$ for each $i \in N$; (ii) for each $\mathbf{r}, \mathbf{r}^{\prime} \in \operatorname{comp}\left\{\mathbf{r}^{*}, \mathbf{e}^{1}, \ldots, \mathbf{e}^{n}\right\}$, for each $i \in N, C_{i}^{r_{i}}=C_{i}^{r_{i}^{\prime}}$ holds if $r_{i}=r_{i}^{\prime}$ and $C_{i}^{r_{i}}>C_{i}^{r_{i}^{\prime}}$ if $r_{i}>r_{i}^{\prime}$; and (iii) for any $\mathbf{r}^{\prime} \in \operatorname{comp}\left\{\mathbf{r}^{*}, \mathbf{e}^{1}, \ldots, \mathbf{e}^{n}\right\}, \lim _{\mathbf{r} \rightarrow \mathbf{r}^{\prime}}\left(C_{i}^{r_{i}}\right)_{i \in N}=\left(C_{i}^{r_{i}^{\prime}}\right)_{i \in N}$ holds. By definition, $G\left(\mathcal{C}_{s}^{\prime \prime}\right)=\operatorname{comp}\left\{\mathbf{r}^{*}, \mathbf{e}^{1}, \ldots, \mathbf{e}^{n}\right\}$.

We now use the information about $\mathcal{C}_{s}^{\prime \prime}$ to construct $\mathbf{e}^{\prime \prime}=\left(M^{\prime \prime}, \widehat{\mathbf{c}}^{1}, \bar{x}^{\prime \prime}\right) \in \mathcal{E}$, in which $M^{\prime \prime} \cap M=\varnothing, \# M^{\prime \prime}=1$, and $\bar{x}^{\prime \prime}=1$. For each $i \in N$, the opportunity correspondence $\widehat{c}_{i}^{1}$ is defined as follows: for all $x \in[0,1]$,

(i) $\widehat{c}_{i}^{1}(1)=C_{i}^{1}, \widehat{c}_{i}^{1}\left(\frac{1}{n}\right)=C_{i}^{r_{i}^{*}}$, and $\widehat{c}_{i}^{1}(0)=\{\mathbf{0}\}$;

(ii) for all $x \in\left(0, \frac{1}{2 n}\right), \widehat{c}_{i}^{1}(x)=\{\mathbf{0}\}$; and

(ii) for all $x \in\left(\frac{1}{2 n}, \frac{1}{n}\right), \widehat{c}_{i}^{1}(x)=C_{i}^{r^{\prime}}$ with $r^{\prime}=2 n\left(x-\frac{1}{2 n}\right) \cdot r_{i}^{*}$; and

(iii) for all $x \in\left(\frac{1}{n}, \frac{n+1}{2 n}\right), \widehat{c}_{i}^{1}(x)=C_{i}^{r_{i}^{*}}$; and

(iv) for all $x \in\left(\frac{n+1}{2 n}, 1\right), \widehat{c}_{i}^{1}(x)=C^{r^{\prime \prime}}$ with $r^{\prime \prime}=\frac{2 n x-(n+1)}{n-1}\left(1-r_{i}^{*}\right)+r_{i}^{*}$.

Then, $\mathcal{C}_{s}^{\prime \prime}=\mathcal{C}\left(\mathbf{e}^{\prime \prime}\right) \in \Sigma$. 
By (WCI), $\mathbf{r}^{*} \in G\left(F\left(\mathcal{C}_{s}^{\prime \prime}\right)\right)$. Next, consider $\mathcal{C}^{\triangle} \equiv \cup_{\pi \in \Pi} \pi\left(\mathcal{C}_{s}^{\prime \prime}\right)$. We will construct a new economy $\mathbf{e}^{\triangle}=\left(M^{\triangle}, \mathbf{c}^{\triangle n}, \bar{x}^{\triangle}\right) \in \mathcal{E}$ such that $\mathcal{C}\left(\mathbf{e}^{\triangle}\right) \supseteq \mathcal{C}^{\triangle}$ and $G\left(\mathcal{C}\left(\mathbf{e}^{\triangle}\right)\right)=G\left(\mathcal{C}^{\triangle}\right)$. First, define an opportunity correspondence $c^{n}$ : $\left[0, \bar{x}^{\prime \prime}\right]^{n} \rightarrow \mathbb{R}_{+}^{k}$ by: for every $x=\left(x_{i}\right)_{i \in N} \in\left[0, \bar{x}^{\prime \prime}\right]^{n}, c^{n}(x) \equiv \cup_{i \in N} \widehat{c}_{i}^{1}\left(x_{i}\right)$. Consider $\mathbf{e}^{(n)}=\left(M^{\prime \prime(n)}, \mathbf{c}^{n},\left(\bar{x}^{\prime \prime}\right)^{n}\right) \in \mathcal{E}$, where $c_{i}^{n}=c^{n}$ for every $i \in N$, and $\left(\bar{x}^{*}\right)^{n} \equiv \underbrace{\left(\bar{x}^{*}, \ldots, \bar{x}^{*}\right)}_{n \text {-times }}$. Then, $\mathcal{C}\left(\mathbf{e}^{(n)}\right) \supseteq \mathcal{C}^{\triangle}$, but $G\left(\mathcal{C}\left(\mathbf{e}^{(n)}\right)\right) \supsetneq G\left(\mathcal{C}^{\triangle}\right)$.

Note that both $G\left(\mathcal{C}\left(\mathbf{e}^{(n)}\right)\right)$ and $G\left(\mathcal{C}^{\triangle}\right)$ are symmetric, and $\partial G\left(\mathcal{C}\left(\mathbf{e}^{(n)}\right)\right)$ is homeomorphic to $\partial G\left(\mathcal{C}^{\triangle}\right)$. Thus, we can take a continuous bijection $\boldsymbol{\lambda}: \partial G\left(\mathcal{C}\left(\mathbf{e}^{(n)}\right)\right) \rightarrow \partial G\left(\mathcal{C}^{\triangle}\right)$ such that for any $\mathbf{r} \in \partial G\left(\mathcal{C}\left(\mathbf{e}^{(n)}\right)\right)$, there exists a scalor $\lambda^{\mathbf{r}} \geq 0$ such that $\boldsymbol{\lambda}(\mathbf{r})=\left(-\lambda^{\mathbf{r}}+r_{i}\right)_{i \in N} \in \partial G\left(\mathcal{C}^{\triangle}\right)$. Define an opportunity correspondence $c^{\triangle n}:\left[0, \bar{x}^{\prime \prime}\right]^{n} \rightarrow \mathbb{R}_{+}^{k}$ as follows: For any $i \in N$, any $\left(-\lambda^{\mathbf{r}}+r_{i}\right)_{i \in N} \in \partial G\left(\mathcal{C}^{\triangle}\right)$, any $r \in\left[0,-\lambda^{\mathbf{r}}+r_{i}\right]$, and any $x \in\left(c^{n}\right)^{-1} \circ$ $G^{-1}(r)$, let $c^{\triangle n}(x)=c^{n}(x)$; and for any $\mathbf{r} \in \partial G\left(\mathcal{C}\left(\mathbf{e}^{(n)}\right)\right)$ and any $i \in N$, if $-\lambda^{\mathbf{r}}+r_{i}<r_{i}$, then for any $r \in\left(-\lambda^{\mathbf{r}}+r_{i}, r_{i}\right]$, and any $x \in\left(c^{n}\right)^{-1} \circ G^{-1}(r)$, let $c^{\triangle n}(x) \in G^{-1}\left(-\lambda^{\mathbf{r}}+r_{i}\right)$. Then, $\mathbf{e}^{\triangle} \equiv\left(M^{*(n)}, \mathbf{c}^{\triangle n},\left(\bar{x}^{*}\right)^{n}\right) \in \mathcal{E}$, where $\mathbf{c}^{\triangle n}=\underbrace{\left(c^{\triangle n}, \ldots, c^{\triangle n}\right)}_{n \text {-times }}$. By definition, $\mathcal{C}\left(\mathbf{e}^{\triangle}\right) \supseteq \mathcal{C}^{\triangle}$, and $G\left(\mathcal{C}\left(\mathbf{e}^{\triangle}\right)\right)=G\left(\mathcal{C}^{\triangle}\right)$.

From the construction of $\mathcal{C}\left(\mathbf{e}^{\triangle}\right),(\mathrm{WE}),(\mathrm{S})$, and (II) imply that

$$
G\left(F\left(\mathcal{C}\left(\mathbf{e}^{\triangle}\right)\right)\right)=\left\{G\left(\mathbf{c}^{\triangle n}\left(\left(\frac{1^{*}}{n}\right)^{n}\right)\right)\right\} .
$$

Let $\mathbf{r}^{\triangle K} \equiv G\left(\mathbf{c}^{\triangle n}\left(\left(\frac{1^{*}}{n}\right)^{n}\right)\right)$, which is the Kalai-Smorodinsky outcome for the problem $\mathcal{C}\left(\mathbf{e}^{\triangle}\right)$. Since $\mathbf{r}^{\triangle K} \in \partial G\left(\mathcal{C}_{s}^{\prime \prime}\right)$, there exists $\mathbf{C}^{\mathbf{r}^{\Delta K}} \in \mathcal{C}_{s}^{\prime \prime}$ such that $G\left(\mathbf{C}^{\mathbf{r}^{\triangle K}}\right)=\mathbf{r}^{\triangle K}$. Thus, by (WCI), $\left\{\mathbf{r}^{\triangle K}\right\}=G\left(F\left(\mathcal{C}_{s}^{\prime \prime}\right)\right)$, which is a desired contradiction, since $\mathbf{r}^{*} \in G\left(F\left(\mathcal{C}_{s}^{\prime \prime}\right)\right)$. Thus, $G(F(\mathcal{C})) \backslash G\left(F^{K}(\mathcal{C})\right)=\varnothing$. By (II), we can conclude that $F=F^{K}$.

\subsection{The Nash solution}

In this subsection, we will provide a characterization of the Nash solution.

Theorem 3: Let the social evaluation of opportunity sets $R$ be homothetic. Then, the Nash solution $F^{N A}$ is the unique solution satisfying (E), (A), (IACC), (CI) and (II).

To prove Theorem 3, we prove the following lemma, Lemma 1, first. 
Lemma 1: Let the social evaluation of opportunity $R$ be given, and $G$ be the real-valued representation of it. Then, for any $\mathcal{C} \in \Sigma$ and any two Pareto efficient points $\overline{\mathbf{G}}^{1}, \overline{\mathbf{G}}^{2} \in \partial G(\mathcal{C})$ which are mutually different, there exists $\mathbf{e}^{\prime}=$ $\left(M^{\prime}, \widetilde{\mathbf{c}}^{m^{\prime}}, \bar{x}^{\prime}\right) \in \mathcal{E}$ such that $\mathcal{C}\left(\mathbf{e}^{\prime}\right)=\mathcal{C}^{\prime}$ and $\mathcal{C}^{\prime}=G^{-1}\left(\operatorname{comp}\left\{\overline{\mathbf{G}}^{1}, \overline{\mathbf{G}}^{2}\right\}\right) \subseteq \mathcal{C}$.

Proof. Given $\mathcal{C} \in \Sigma$ and any $\overline{\mathbf{G}}^{1}, \overline{\mathbf{G}}^{2} \in \partial G(\mathcal{C})$ which are mutually different, let us consider comp $\left\{\overline{\mathbf{G}}^{1}, \overline{\mathbf{G}}^{2}\right\}$. Let an economy $\mathbf{e}=\left(M, \mathbf{c}^{m}, \bar{x}\right) \in \mathcal{E}$ be such that $\mathcal{C}(\mathbf{e})=\mathcal{C}$. Let $\mathcal{C}^{\prime} \equiv G^{-1}\left(\operatorname{comp}\left\{\overline{\mathbf{G}}^{1}, \overline{\mathbf{G}}^{2}\right\}\right)$. By definition, $\mathcal{C}^{\prime} \subseteq \mathcal{C}$. Given this $\mathcal{C}^{\prime}$, let us define

$$
\mathcal{C}^{\prime}(i) \equiv\left\{C_{i}^{\prime} \in \mathcal{K} \mid \exists \mathbf{C}_{-i} \in \mathcal{K}^{n-1}:\left(C_{i}^{\prime}, \mathbf{C}_{-i}\right) \in \mathcal{C}^{\prime}\right\}
$$

for each $i \in N$. Then, for each $r \geq 0$, define

$$
\mathcal{C}^{\prime}(i ; r) \equiv\left\{C_{i}^{\prime} \in \mathcal{C}^{\prime}(i) \mid G\left(C_{i}^{\prime}\right)=r\right\}
$$

for each $i \in N$. Since $G$ is continuous, we can choose a continuous sequence $\mathcal{C}_{s}^{\prime} \subseteq \mathcal{C}^{\prime}$ in which (i) for each $\mathbf{r} \in \operatorname{comp}\left\{\overline{\mathbf{G}}^{1}, \overline{\mathbf{G}}^{2}\right\}$, there exists a unique $\left(C_{i}^{r_{i}}\right)_{i \in N} \in \mathcal{C}_{s}^{\prime}$ such that $C_{i}^{r_{i}} \in \mathcal{C}^{\prime}\left(i ; r_{i}\right)$ for each $i \in N$; (ii) for each $\mathbf{r}, \mathbf{r}^{\prime} \in$ $\operatorname{comp}\left\{\overline{\mathbf{G}}^{1}, \overline{\mathbf{G}}^{2}\right\}$, for each $i \in N, C_{i}^{r_{i}}=C_{i}^{r_{i}^{\prime}}$ holds if $r_{i}=r_{i}^{\prime}$ and $C_{i}^{r_{i}}>C_{i}^{r_{i}^{\prime}}$ if $r_{i}>r_{i}^{\prime}$; and (iii) for any $\mathbf{r}^{*} \in \operatorname{comp}\left\{\overline{\mathbf{G}}^{1}, \overline{\mathbf{G}}^{2}\right\}, \lim _{\mathbf{r} \rightarrow \mathbf{r}^{*}}\left(C_{i}^{r_{i}}\right)_{i \in N}=\left(C_{i}^{r_{i}^{*}}\right)_{i \in N}$ holds. By definition, $G\left(\mathcal{C}_{s}^{\prime}\right)=\operatorname{comp}\left\{\overline{\mathbf{G}}^{1}, \overline{\mathbf{G}}^{2}\right\}$.

We now construct $\mathbf{e}^{\prime}=\left(M^{\prime}, \overline{\mathbf{c}}^{1}, \bar{x}^{\prime}\right) \in \mathcal{E}$, in which $\# M^{\prime}=1$ and $\bar{x}^{\prime}=1$. Let us denote the $n$-1-dimensional simplex by $\triangle$. This simplex is interpreted as the set of Pareto efficient allocations of the one dimensional commodity $\bar{x}^{\prime}=1$. Each vertex $e_{i} \in \triangle$ corresponds to the allocation that $\bar{x}^{\prime}$ is assigned to individual $i \in N$ and any other individual gets nothing. Now, take a continuous bijection $\boldsymbol{\lambda}: \partial G\left(\mathcal{C}_{s}^{\prime}\right) \rightarrow \triangle$ such that for any $\mathbf{r} \in \partial G\left(\mathcal{C}_{s}^{\prime}\right)$, there exists a scalor $\lambda^{\mathbf{r}}>0$ such that $\boldsymbol{\lambda}(\mathbf{r})=\left(\lambda^{\mathbf{r}} \cdot r_{i}\right)_{i \in N} \in \triangle$. With the help of this mapping, $\overline{\mathbf{c}}^{1}$ of the underlying economy $\mathbf{e}^{\prime}=\left(M^{\prime}, \overline{\mathbf{c}}^{1}, \bar{x}^{\prime}\right) \in \mathcal{E}$ is defined as follows: for each $i \in N, \bar{c}_{i}^{1}$ is such that for all $x \in[0,1], \bar{c}_{i}^{1}(x)=C_{i}^{r_{i}}$ whenever $r_{i}=\left(\lambda^{\mathbf{r}}\right)^{-1}(x)$. Then, $\mathcal{C}\left(\mathbf{e}^{\prime}\right)=\mathcal{C}_{s}^{\prime} \in \Sigma$ holds. By definition, $G\left(\mathcal{C}\left(\mathbf{e}^{\prime}\right)\right)=\operatorname{comp}\left\{\overline{\mathbf{G}}^{1}, \overline{\mathbf{G}}^{2}\right\}$.

Proof of Theorem 3: It can be checked that $F^{N A}$ satisfies the five axioms of the theorem. We need only to show that if a solution $F$ satisfies the five axioms specified in Theorem 3 , then $F=F^{N A}$. 
Let $F$ be a solution satisfying the five axioms of Theorem 3 . Let $G: \mathcal{K} \rightarrow$ $\mathbb{R}_{+}$cardinally represent the homothetic ordering $R$ so that $G$ is homogeneous of degree $l$ for some positive integer $l$. Consider any bargaining problem $\mathcal{C} \in \Sigma$ that is derived from an underlying economic environment $\mathbf{e}=\left(M, \mathbf{c}^{m}, \bar{x}\right) \in \mathcal{E}$. Consider the following problem:

$$
\max _{\left(C_{i}\right)_{i \in N} \in \mathcal{C}} \prod_{i \in N} G\left(C_{i}\right) .
$$

The set of the solutions for the above problem constitutes the Nash-solution set $F^{N A}(\mathcal{C})$ for $\mathcal{C}$.

Suppose $F \neq F^{N A}$. By (II), we can suppose that $G(F(\mathcal{C})) \neq G\left(F^{N A}(\mathcal{C})\right)$ for this $\mathcal{C} \in \Sigma$. Then, either (i) there exists $\overline{\mathbf{G}}^{*} \in G(F(\mathcal{C})) \backslash G\left(F^{N A}(\mathcal{C})\right)$ or (ii) $G(F(\mathcal{C})) \subsetneq G\left(F^{N A}(\mathcal{C})\right)$.

Consider case (i) first. Given the bargaining problem $\mathcal{C}$, we define $\mathcal{C}(i) \equiv$ $\left\{C_{i}^{\prime} \in \mathcal{K} \mid \exists \mathbf{C}_{-i} \in \mathcal{K}^{n-1}:\left(C_{i}^{\prime}, \mathbf{C}_{-i}\right) \in \mathcal{C}\right\}$ for each $i \in N$. Let $\overline{\mathbf{G}}^{N A} \in G\left(F^{N A}(\mathcal{C})\right)$ and $\overline{\mathbf{G}}^{*} \in G(F(\mathcal{C})) \backslash G\left(F^{N A}(\mathcal{C})\right)$. Then, by Lemma 1 , we can construct a new economy $\mathbf{e}^{\prime}=\left(M^{\prime}, \widetilde{\mathbf{c}}^{1}, \bar{x}^{\prime}\right) \in \mathcal{E}$ such that $\mathcal{C}\left(\mathbf{e}^{\prime}\right)=G^{-1}\left(\operatorname{comp}\left\{\overline{\mathbf{G}}^{*}, \overline{\mathbf{G}}^{N A}\right\}\right) \subseteq$ $\mathcal{C}$. Let $\mathcal{C}^{\prime} \equiv \mathcal{C}\left(\mathbf{e}^{\prime}\right)$. By $(\mathrm{CI}), \overline{\mathbf{G}}^{*} \in G\left(F\left(\mathcal{C}^{\prime}\right)\right)$. Note that since $\prod_{i \in N} \bar{G}_{i}^{*}<$ $\prod_{i \in N} \bar{G}_{i}^{N A}$, there exists $\boldsymbol{\varepsilon} \in \mathbb{R}_{+}^{n} \backslash\{\mathbf{0}\}$ such that $\prod_{i \in N}\left(\bar{G}_{i}^{*}+\varepsilon_{i}\right)=\prod_{i \in N} \bar{G}_{i}^{N A}$. Let $\mathbf{r}^{* *} \equiv \overline{\mathbf{G}}^{*}+\boldsymbol{\varepsilon}$. Since $\mathbf{r}^{* *} \leq \mathbf{m}(G(\mathcal{C}))$, there exists a profile of opportunity $\mathbf{C}^{\mathbf{r}^{* *}} \in \mathcal{K}^{n}$ such that for each $i \in N, C_{i}^{r_{i}^{* *}} \in \mathcal{C}(i), G\left(C_{i}^{r_{i}^{* *}}\right)=r_{i}^{* *}$, and $C_{i}^{r_{i}^{* *}} \supseteq C_{i}^{\bar{G}_{i}^{*}}$, where $C_{i}^{\bar{G}_{i}^{*}}=G^{-1}\left(\bar{G}_{i}^{*}\right)$. Then, we can construct another economy $\mathbf{e}^{\prime \prime}=\left(M^{\prime \prime}, \widehat{\mathbf{c}}^{1}, \bar{x}^{\prime \prime}\right) \in \mathcal{E} \operatorname{such}$ that $\mathcal{C}\left(\mathbf{e}^{\prime \prime}\right)=G^{-1}\left(\operatorname{comp}\left\{\mathbf{r}^{* *}, \overline{\mathbf{G}}^{N A}\right\}\right) \supseteq$ $\mathcal{C}^{\prime} \cup\left\{\mathbf{C}^{\mathbf{r}^{* *}}\right\}$.

Given that $\prod_{i \in N}\left(r_{i}^{* *}\right)=\prod_{i \in N} \bar{G}_{i}^{N A}$, we choose the $n$ dimensional positive vector $\mathbf{a}=\left(a_{i}\right)_{i \in N} \in \mathbb{R}_{++}^{n}$ appropriately as in the proof of Theorem 1 in Xu and Yoshihara (2006), so that $\left(a_{i} r_{i}^{* *}\right)_{i \in N}=\pi^{0}\left(\left(a_{i} \bar{G}_{i}^{N A}\right)_{i \in N}\right)$ and $\pi^{0}\left(\left(a_{i} r_{i}^{* *}\right)_{i \in N}\right)=\left(a_{i} \bar{G}_{i}^{N A}\right)_{i \in N}$ for some permutation $\pi^{0} \in \Pi$. By homotheticity of $R, \mathbf{e}^{\prime \prime \prime}=\left(M^{\prime \prime}, \mathbf{a}^{\frac{1}{l}} \cdot \widehat{\mathbf{c}}^{1}, \bar{x}^{\prime \prime}\right) \in \mathcal{E}$ constitutes $\mathcal{C}^{\prime \prime \prime} \equiv \mathcal{C}\left(\mathbf{e}^{\prime \prime \prime}\right)=$ $G^{-1}\left(\operatorname{comp}\left\{\mathbf{a} \cdot \mathbf{r}^{* *}, \mathbf{a} \cdot \overline{\mathbf{G}}^{N A}\right\}\right)$.

Next, consider $\mathcal{C}^{\triangle} \equiv \cup_{\pi \in \Pi} \pi\left(\mathcal{C}^{\prime \prime \prime}\right)$. We will construct a new economy $\mathbf{e}^{\triangle}=\left(M^{\triangle}, \mathbf{c}^{\triangle n}, \bar{x}^{\triangle}\right) \in \mathcal{E}$ such that $\mathcal{C}\left(\mathbf{e}^{\triangle}\right) \supseteq \mathcal{C}^{\triangle}$ and $G\left(\mathcal{C}\left(\mathbf{e}^{\triangle}\right)\right)=G\left(\mathcal{C}^{\triangle}\right)$. 
First, define an opportunity correspondence $c^{n}:\left[0, \bar{x}^{\prime \prime}\right]^{n} \rightarrow \mathbb{R}_{+}^{k}$ by: for every $x=\left(x_{i}\right)_{i \in N} \in\left[0, \bar{x}^{\prime \prime}\right]^{n}, c^{n}(x) \equiv \cup_{i \in N} a_{i}^{\frac{1}{l}} \widehat{c}_{i}^{1}\left(x_{i}\right)$. Let us consider $\mathbf{e}^{(n)}=$ $\left(M^{\prime \prime}(n), \mathbf{c}^{n},\left(\bar{x}^{\prime \prime}\right)^{n}\right) \in \mathcal{E}$, where $c_{i}^{n}=c^{n}$ for every $i \in N$, and $\left(\bar{x}^{\prime \prime}\right)^{n} \equiv \underbrace{\left(\bar{x}^{\prime \prime}, \ldots, \bar{x}^{\prime \prime}\right)}_{n \text {-times }}$.

Then, $\mathcal{C}\left(\mathbf{e}^{(n)}\right) \supseteq \mathcal{C}^{\triangle}$, but $G\left(\mathcal{C}\left(\mathbf{e}^{(n)}\right)\right) \supsetneq G\left(\mathcal{C}^{\triangle}\right)$. Note that both $G\left(\mathcal{C}\left(\mathbf{e}^{(n)}\right)\right)$ and $G\left(\mathcal{C}^{\triangle}\right)$ are symmetric, and $\partial G\left(\mathcal{C}\left(\mathbf{e}^{(n)}\right)\right)$ is homeomorphic to $\partial G\left(\mathcal{C}^{\triangle}\right)$. Thus, we can take a continuous bijection $\boldsymbol{\lambda}: \partial G\left(\mathcal{C}\left(\mathbf{e}^{(n)}\right)\right) \rightarrow \partial G\left(\mathcal{C}^{\triangle}\right)$ such that for any $\mathbf{r} \in \partial G\left(\mathcal{C}\left(\mathbf{e}^{(n)}\right)\right)$, there exists a scalor $\lambda^{\mathbf{r}}>0$ such that $\boldsymbol{\lambda}(\mathbf{r})=\left(\lambda^{\mathbf{r}} \cdot r_{i}\right)_{i \in N} \in \partial G\left(\mathcal{C}^{\triangle}\right)$. Define an opportunity correspondence $c^{\triangle n}$ as follows: For any $\left(\lambda^{\mathbf{r}} \cdot r_{i}\right)_{i \in N} \in \partial G\left(\mathcal{C}^{\triangle}\right)$, any $i \in N$, any $r \in\left[0, \lambda^{\mathbf{r}} \cdot r_{i}\right]$, and any $x \in\left(c^{n}\right)^{-1} \circ G^{-1}(r)$, let $c^{\triangle n}(x)=c^{n}(x)$; and for any $\mathbf{r} \in \partial G\left(\mathcal{C}\left(\mathbf{e}^{(n)}\right)\right)$, if $\lambda^{\mathbf{r}} \cdot r_{i}<r_{i}$, then for any $r \in\left(\lambda^{\mathbf{r}} \cdot r_{i}, r_{i}\right]$, and any $x \in\left(c^{n}\right)^{-1} \circ G^{-1}(r)$, let $c^{\triangle n}(x) \in G^{-1}\left(\lambda^{\mathbf{r}} \cdot r_{i}\right)$. Then, $\mathbf{e}^{\triangle} \equiv\left(M^{\prime \prime(n)}, \mathbf{c}^{\triangle n},\left(\bar{x}^{\prime \prime}\right)^{n}\right) \in \mathcal{E}$, where $\mathbf{c}^{\triangle n}=\underbrace{\left(c^{\triangle n}, \ldots, c^{\triangle n}\right)}_{n \text {-times }}$. By definition, $\mathcal{C}\left(\mathbf{e}^{\triangle}\right) \supseteq \mathcal{C}^{\triangle}$, and $G\left(\mathcal{C}\left(\mathbf{e}^{\triangle}\right)\right)=G\left(\mathcal{C}^{\triangle}\right)$.

From the construction of $\mathcal{C}\left(\mathbf{e}^{\triangle}\right),(\mathrm{E})$ and $(\mathrm{A})$ imply that

$$
\left\{\mathbf{a} \cdot \mathbf{r}^{* *}, \mathbf{a} \cdot \overline{\mathbf{G}}^{N A}\right\} \cup\left\{\pi\left(\mathbf{a} \cdot \mathbf{r}^{* *}\right), \pi\left(\mathbf{a} \cdot \overline{\mathbf{G}}^{N}\right)\right\}_{\pi \in \Pi}=G\left(F\left(\mathcal{C}\left(\mathbf{e}^{\triangle}\right)\right)\right) .
$$

Thus, by (CI), $\left\{\mathbf{a} \cdot \mathbf{r}^{* *}, \mathbf{a} \cdot \overline{\mathbf{G}}^{N A}\right\}=G\left(F\left(\mathcal{C}^{\prime \prime \prime}\right)\right)$. By (IACC), $\left\{\mathbf{r}^{* *}, \overline{\mathbf{G}}^{N A}\right\}=$ $G\left(F\left(\mathcal{C}\left(\mathbf{e}^{\prime \prime}\right)\right)\right)$. By $(\mathrm{CI}),\left\{\overline{\mathbf{G}}^{N A}\right\}=G\left(F\left(\mathcal{C}^{\prime}\right)\right)$, a contradiction since $\overline{\mathbf{G}}^{*} \in$ $G\left(F\left(\mathcal{C}^{\prime}\right)\right)$. Therefore, $G(F(\mathcal{C})) \backslash G\left(F^{N A}(\mathcal{C})\right)=\varnothing$.

Now, consider case (ii). Since $G(F(\mathcal{C})) \subsetneq G\left(F^{N A}(\mathcal{C})\right)$, by (II), we can suppose that $\left\{\mathbf{r}^{* *}, \overline{\mathbf{G}}^{N A}\right\} \subseteq G\left(F^{N A}(\mathcal{C})\right)$, but $\overline{\mathbf{G}}^{N A} \notin G(F(\mathcal{C}))$. Then, consider comp $\left\{\mathbf{r}^{* *}, \overline{\mathbf{G}}^{N A}\right\}$. Following a similar proof method as for case (i), we can derive another contradiction.

The two cases (i) and (ii) exhaust all possibilities. Therefore, it is true that $F=F^{N A}$.

\subsection{Interpretations}

Structurally, our axioms and solutions are similar to those of standard bargaining problems. However, we believe that interpretations of our results are quite different from those of standard bargaining models. 
First, in the standard bargaining model with individual utilities, a solution can be regarded as the recommendation from the viewpoint of equality of resources (Dworkin (1981)), as discussed in Roemer $(1986,1988)$ and in Yoshihara $(2003,2006)^{5}$. On the other hand, as we already discussed in the Introduction, a solution in our context is closely linked with the principle of equality of opportunity (Roemer (1998)). The principle of equal opportunity is to "equalize" the playing fields for all individuals as much as possible (Roemer (1998)), and each of our three solutions embodies this idea.

Secondly, implications of each of our three solutions on equal opportunity mechanisms can be very different. For instance, Roemer (1998) argues that an optimal equal opportunity policy should guarantee equal opportunity of accessing to the same level of "advantage" regardless of their "types" for any two individuals, as long as their effort ranking within their own "types" are identical. In our model, we may interpret an individual's type in the sense of Roemer as reflected in the individual's opportunity correspondence when the difference in individuals' opportunity correspondences is due mainly to the difference in individuals' native talents or circumstances. In such a context, the egalitarian solution seems the most reasonable solution based on the principle of equal opportunity since it guarantees an "equal" assignment of opportunities among individuals.

However, when the difference in individuals' opportunity correspondences is also based on the difference in their supply of effort levels, the egalitarian solution may no longer be the most reasonable solution in our context. This is because it ignores the responsibility aspect (Fleurbaey (1998)). For example, when there is an enlargement or a shrinkage of an individual's opportunity set, we want to find out whether or not the individual is responsible for such a change of his opportunity sets. When such information becomes available and responsibility factors are identifiable, the Nash and Kalai-Smorodinsky solutions are more reasonable than the egalitarian solution, since responsibility and compensation may be captured by, respectively, the axiom IACC and contraction-type axoims, and both the Nash solution

\footnotetext{
${ }^{5}$ Yoshihara $(2003,2006)$ shows that the Nash solution is the most plausible bargaining solution for the Dworkinian resource egalitarianism if one uses the principle of responsibility and compensation as discussed in Fleurbaey (1998). According to Yoshihara (2003, 2006), the scale invariance axiom in the standard bargaining model with individual utilities can be regarded as an axiom of responsibility for individual utility functions, while the monotonicity type axiom and the contraction independence axiom can be seen as axioms of compensation.
} 
and the Kalai-Smorodinsky solution satisfy IACC and various versions of contraction axioms.

\subsection{Subjective Measures of Opportunity Sets}

So far, we have assumed that there is an objective measure of opportunity sets across individuals. One may argue that the objective measure is "too paternalistic" and one should allow possibilities of self-evaluations of opportunity sets by individuals. Thus, for each individual $i \in N$, there is an ordering $R_{i}$ over the set of all opportunity sets. If we adopt this approach, we can obtain similar characterization results of the Nash and the Kalai-Smorodinsky solutions by making following observations.

For each $i \in N$, let $G_{i}$ be a real-valued function representing $i$ 's personal evaluation, $R_{i}$, of opportunity sets satisfying Monotonicity, Cardinal Representability and Homotheticity. For each $i \in N$, let $I_{i}$ stand for the symmetric part of $R_{i}$. Consider now the following axiom:

S-Informational Invariance (SII): For each $\mathcal{C} \in \Sigma$ and each $\mathbf{C}=\left(C_{i}\right)_{i \in N}, \mathbf{C}^{\prime}=$ $\left(C_{i}^{\prime}\right)_{i \in N} \in \mathcal{C}$, if $\mathbf{C} \in F(\mathcal{C})$ and $\left(C_{i}^{\prime}, C_{i}\right) \in I_{i}$ for all $i \in N$, then $\mathbf{C}^{\prime} \in F(\mathcal{C})$.

Therefore, according to (SII), if two opportunity assignments are "equivalent" in the sense that the two opportunity sets for each and every individual specified by the corresponding opportunity assignments are ranked equally according to their subjective evaluation of opportunity sets, then whenever one opportunity assignment is in the solution, the other should be in as well.

With the possibility of personal evaluation of opportunity sets and the above axiom (SII), we can obtain the following results. The proofs of them are similar to those of Theorems 2 and 3 and we therefore omit them.

Theorem 4: Let each $R_{i}(i \in N)$ be homothetic. Then, the Kalai-Smorodinsky solution $F^{K}$ is the unique solution satisfying (WE), (S), (IACC), (WCI) and (SII).

Theorem 5: Let each $R_{i}(i \in N)$ be homothetic. Then, the Nash solution $F^{N A}$ is the unique solution satisfying (E), (A), (IACC), (CI) and (SII). 


\section{Conclusion}

In this paper, we have extended the standard bargaining model to situations in which players are characterized by their opportunity sets rather than by their utilities and in which the fair arbitrator makes the recommendation with the guiding principle of equal opportunity for all players. In such a setting, we have formulated our problems in terms of bargaining problems among players on opportunity assignments, defined several solution concepts such as the Nash solution, the egalitarian solution and the Kalai-Smorodinsky solution in our contex and studied them axiomatically. Most of the axioms used in our axiomatic characterizations of the proposed solutions are their counterparts in standard bargaining models, but formulated in terms of opportunity assignments. We have discussed and commented on the axioms that are unique in our context.

We should mention that, in our characterizations of the Nash and the Kalai-Smorodinsky solutions, we assume that the function $G$ is homothetic. As we argued in Section 3, in our context, the homotheticity of $G$ is a reasonable property. Nevertheless, if one abandons this property on $G$, we can obtain similar characterizations of the Nash and the Kalai-Smorondinsky solutions by replacing (IACC) with the following axiom:

Modified Invariance to Affine Changes in opportunity Correspondences (MIACC): For each $\mathcal{C}, \mathcal{C}^{\prime} \in \Sigma$, if there exists a vector $\mathbf{a}=\left(a_{i}\right)_{i \in N} \in$ $\mathbb{R}_{++}^{n}$ such that $\mathcal{C}^{\prime}=\left\{\left(C_{i}^{\prime}\right)_{i \in N} \subseteq \mathbb{R}_{+}^{k n} \mid G\left(C_{i}^{\prime}\right)=a_{i} G\left(C_{i}\right), i \in N, \mathbf{C} \in \mathcal{C}\right\}$, then $\mathbf{C} \in F(\mathcal{C}) \Leftrightarrow \mathbf{C}^{\prime} \in F\left(\mathcal{C}^{\prime}\right)$ whenever $G\left(C_{i}^{\prime}\right)=a_{i} G\left(C_{i}\right)$ for all $i \in N$.

(MIACC) has a similar intuition as that of (IACC), and is a reasonable property given that $G$ is cardinal.

Our approach is, we believe, a first attempt to extend the standard bargaining model to a setting in which individuals are characterized by their opportunity sets, and opens a new avenue for understanding bargaining problems.

\section{References}

1. Arneson, R. (1989): "Equality and equal opportunity for welfare," Philosophical Studies 56, 77-93. 
2. Cohen, G.A. (1993): "Equality of what? On welfare, goods, and capabilities," in The Quality of Life, (edited by Nussbaum, M. and A. Sen), Oxford Univ. Press: Oxford, 9-29.

3. Dworkin, R. (1981): "What is equality ? Part 2: equality of resources," Philosophy $\&$ Public Affairs 10, 283-345.

4. Fleurbaey, M. (1998): "Equality among responsible individuals," in Freedom in Economics, (edited by Laslier, J-F., Fleurbaey, M., Gravel, N., and A. Trannoy), Routledge: New York, 25-55.

5. Gaertner, W. and Y. Xu (2006): "Capability as the Basis for a New Measure of the Standard of Living," Journal of Human Development 7(3), 311-321.

6. Gorman, W. (1980): "The demand for related goods: a possible procedure for analysing quality differentials in the egg market," Review of Economic Studies 47, 843-856.

7. Gotoh, R. and N. Yoshihara (2003): "A class of fair distribution rules a la Rawls and Sen," Economic Theory 22, 63-88.

8. Herrero, C. (1996): "Capabilities and utilities," Economic Design 2, 69-88.

9. Herrero, C. (1997): "Equitable opportunities: An extension," Economics Letters 55, 91-95.

10. Kranich, L. (1996): "Equitable opportunities: An axiomatic approach," Journal of Economic Theory 71, 131-147.

11. Lancaster, K. J., (1966): "A new approach to consumer theory," Journal of Political Economy 74, 132-57.

12. Nash, J. F. (1950): “The bargaining problem," Econometrica, 18, 155162.

13. Nussbaum, M. (1988): "Nature, function and capability: Aristotle on political distribution," Oxford Studies in Ancient Philosophy, I (supplementary), 145-84. 
14. Nussbaum, M. (1993): "Non-relative virtues: an Aristotelian approach", in M. Nussbaum and A.K. Sen (eds.), The Quality of Life, Clarendon Press, Oxford.

15. Nussbaum, M. (2000): Women and Human Development: The Capability Approach, Kali for Women, New Delhi.

16. Pattanaik, P.K. and Y. Xu (1990): "On ranking opportunity sets in terms of freedom of choice," Recherches Economiques de Louvain, 56, 383-390.

17. Pattanaik, P.K. and Y. Xu (2000): "On ranking opportunity sets in economic environments," Journal of Economic Theory 93(1), 48-71.

18. Pattanaik, P.K. and Y. Xu (2006): "Minimal relativism, dominance, and standard of living comparisons based on functionings," Oxford Economic Papers, forthcoming.

19. Roemer, J. E., (1986): "Equality of resources implies equality of welfare," Quarterly Journal of Economics 101, 751-784.

20. Roemer, J. E., (1988): "Axiomatic bargaining theory on economic environments," Journal of Economic Theory 45, 1-31.

21. Roemer, J.E. (1998): Equality of opportunity, Harvard Univ. Press: Cambridge.

22. Savaglio, E. and S. Vannucci (2006): "On the volume-ranking of opportunity sets in economic environments," mimeo.

23. Sen, A. K. (1980): "Equality of what?" in Tanner Lectures on Human Values. Vol. 1, (edited by McMurrin, S.), Cambridge Univ. Press: Cambridge.

24. Sen, A. K. (1985): Commodities and capabilities, Amsterdam: NorthHolland.

25. Sen, A. K. (2002): Rationality and freedom, Harvard University Press: Cambridge, MA.

26. Thomson, W. (1983): "Equity in exchange economies," Journal of Economic Theory 29, 217-244. 
27. Xu, Y. (2002): "Functioning, capability, and the standard of living - an axiomatic approach," Economic Theory 20, 387-399.

28. Xu, Y. (2004): "On ranking linear budget sets in terms of freedom of choice," Social Choice and Welfare 22(1), 281-289.

29. Xu, Y. and N. Yoshihara (2006): "Alternative characterizations of three bargaining solutions for nonconvex problems," Games and Economic Behaviors 57, 86-92.

30. Yoshihara, N., (2003): "Characterizations of bargaining solutions in production economies with unequal skills," Journal of Economic Theory 108, 256-285.

31. Yoshihara, N., (2006): "Solidarity and cooperative bargaining solutions," in A. Wieczorek, M. Malawski, and A. Wiszniewska-Matyszkiel, eds., Game Theory and Mathematical Economics, Banach Center Publications 70, Warszawa, pp. 317-330. 


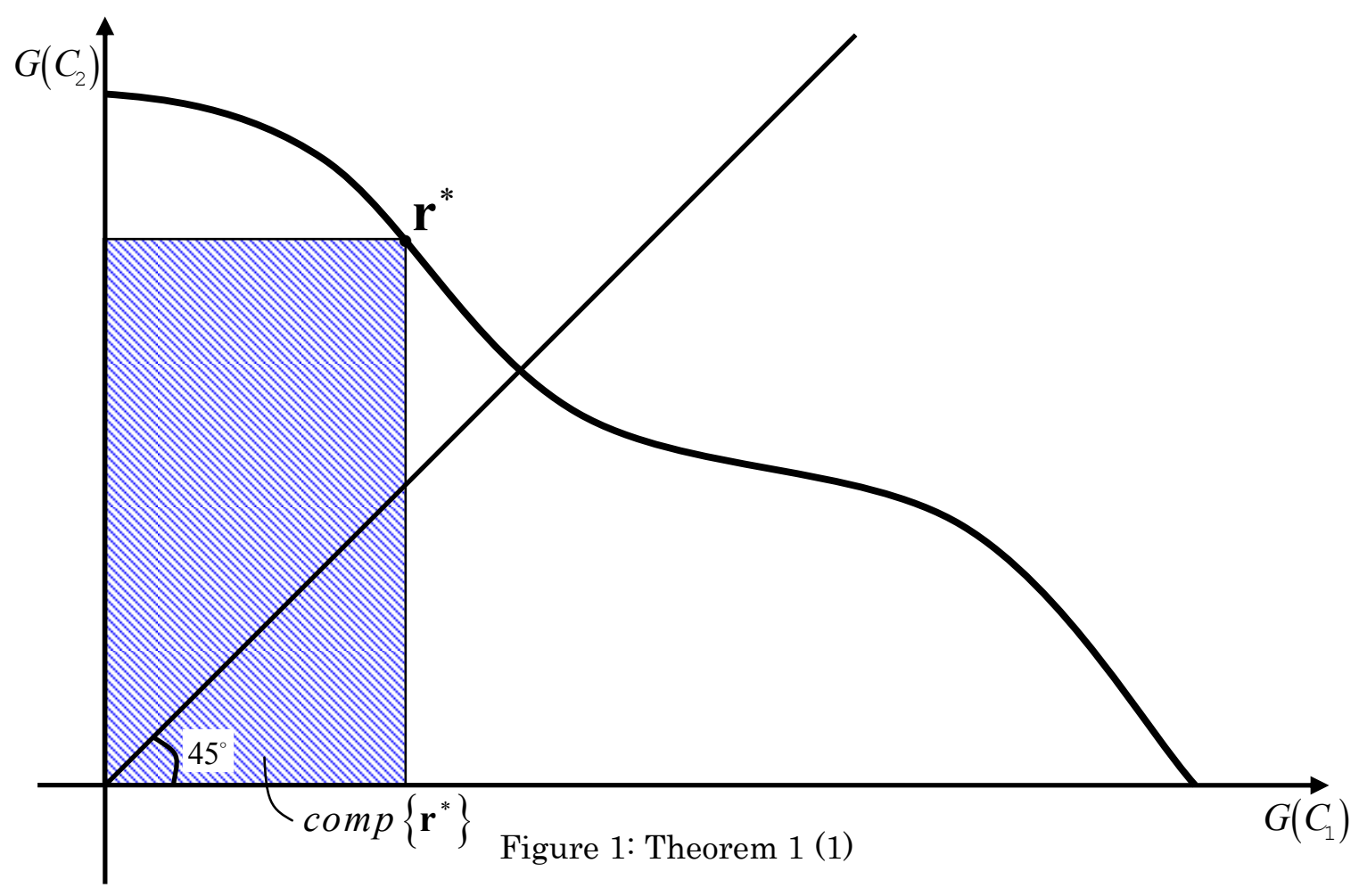




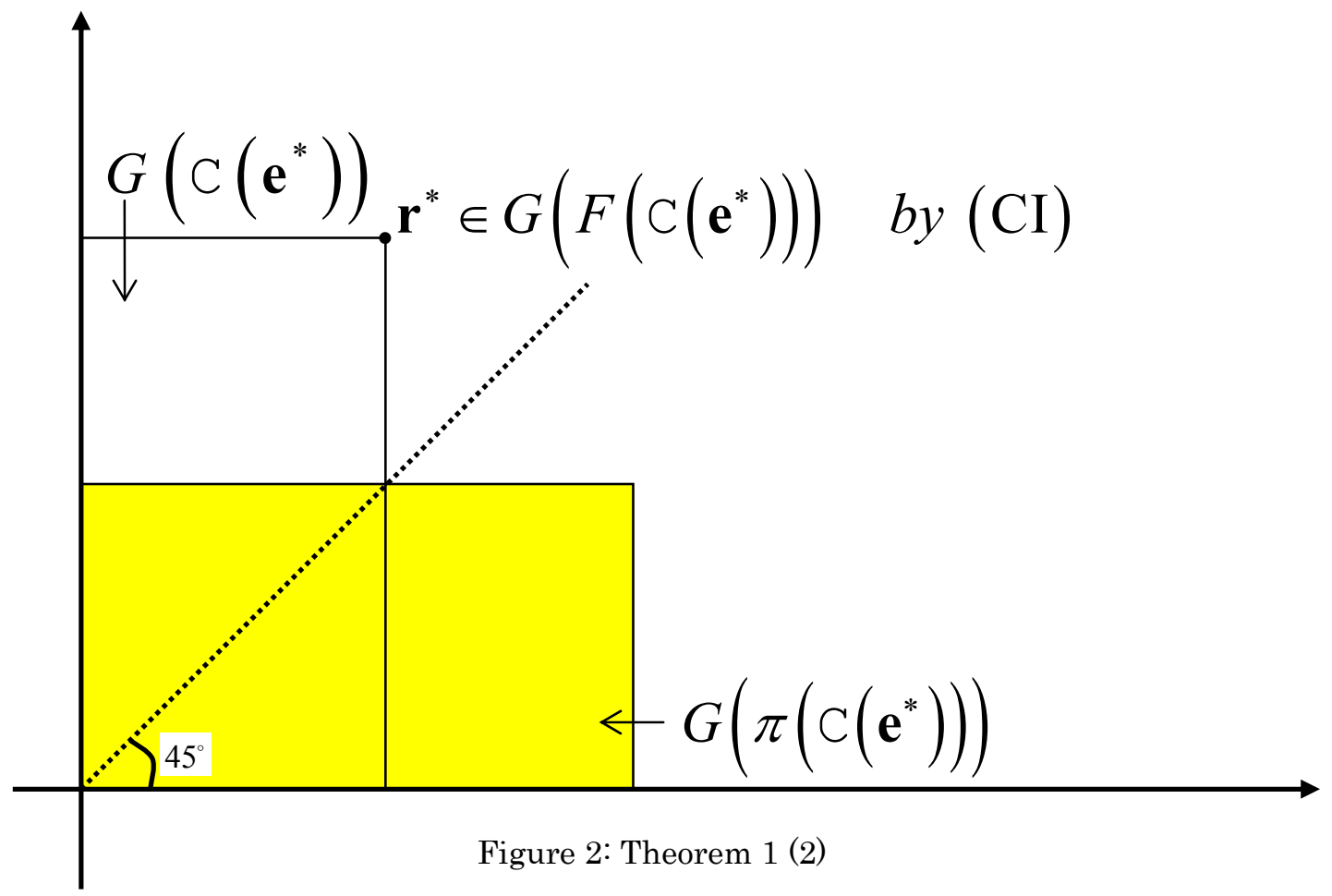




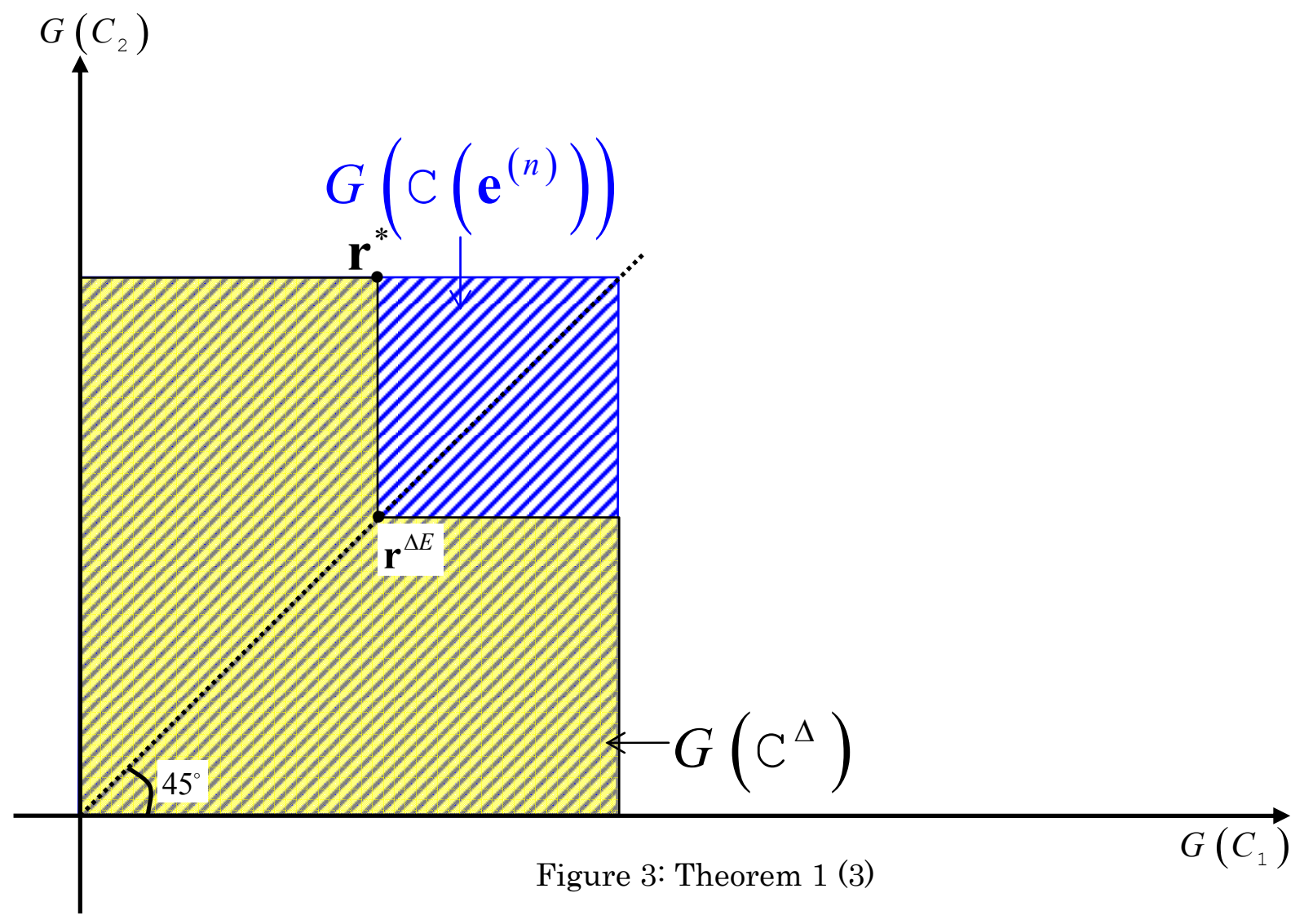




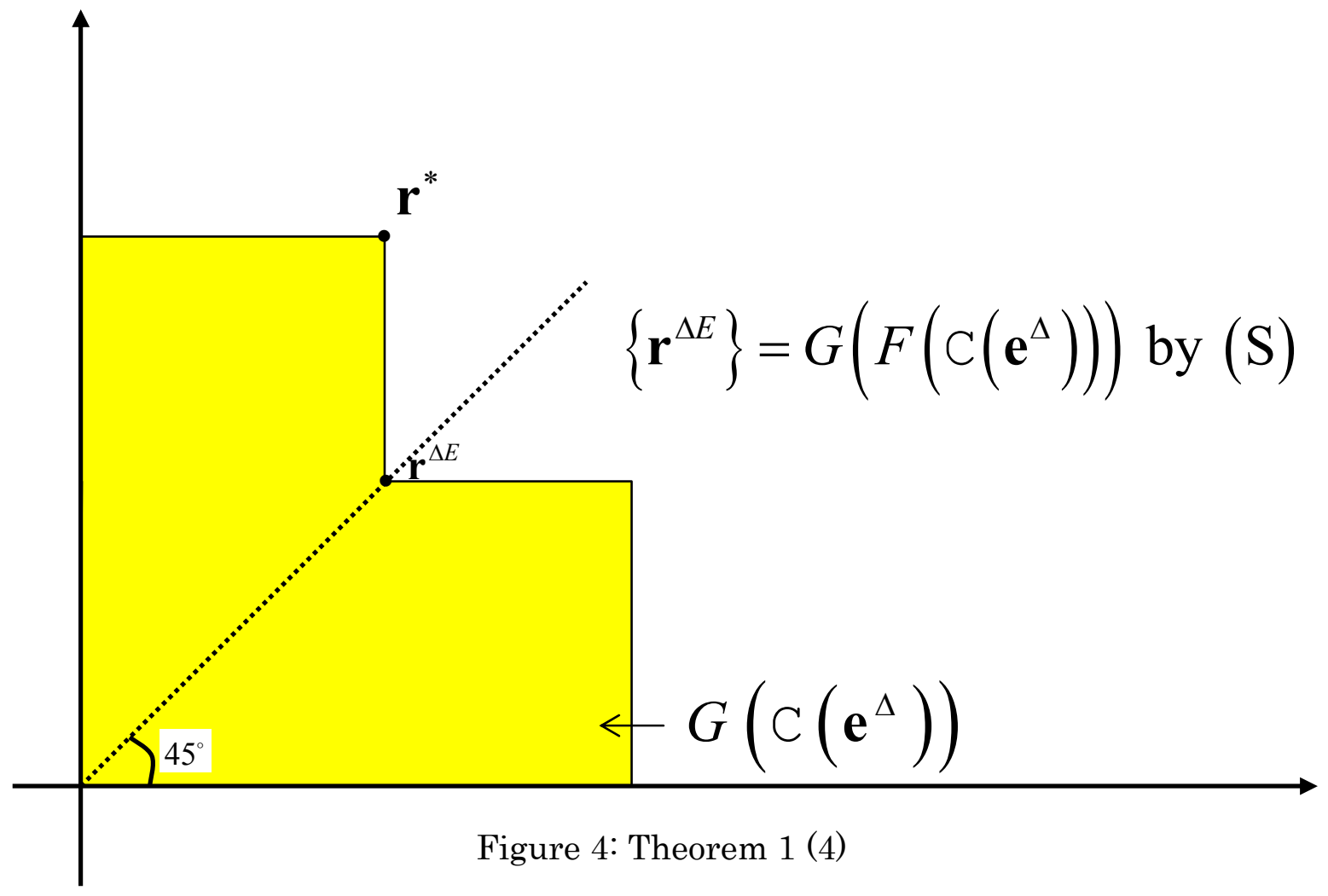




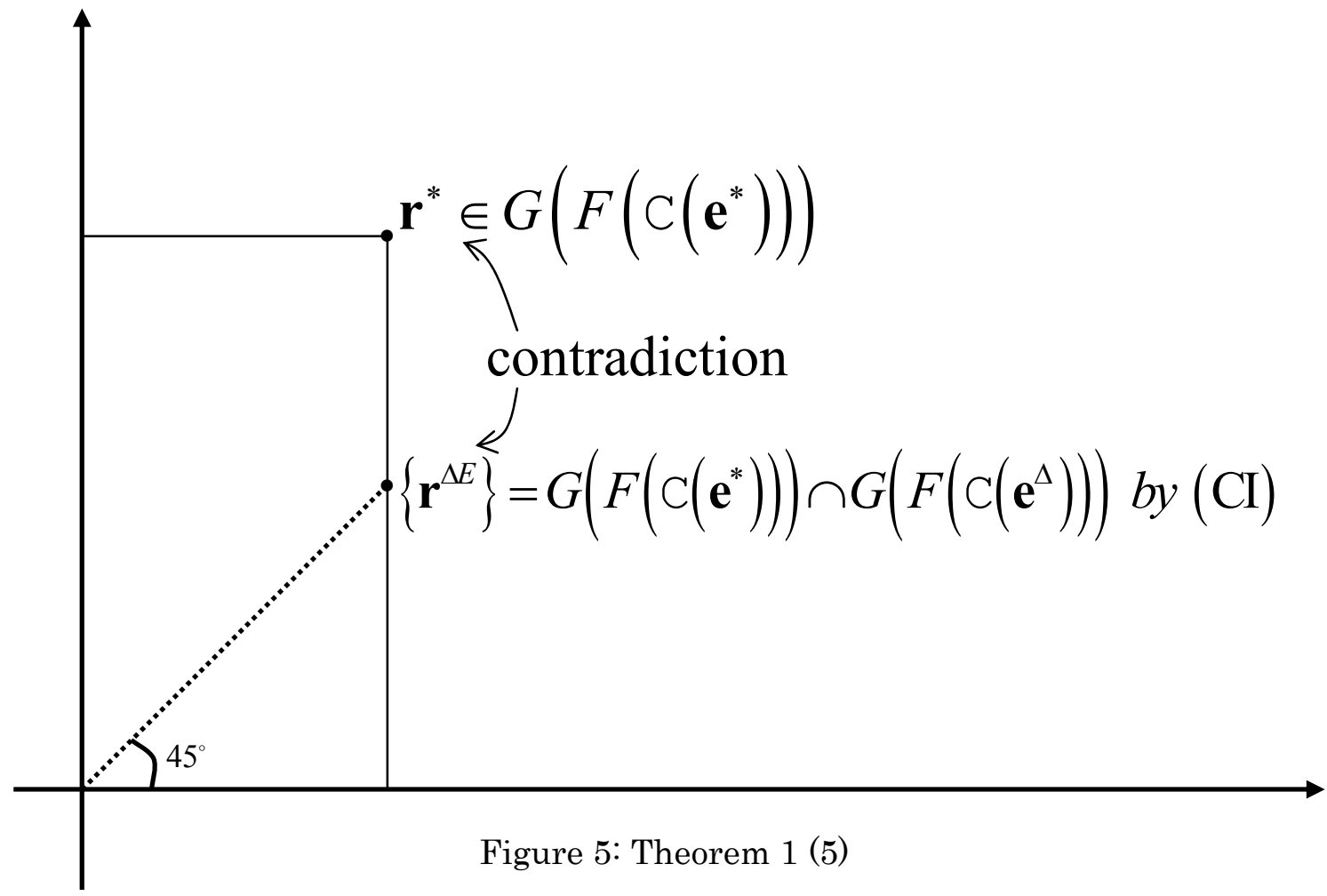

